\title{
A new renormalon in two dimensions
}

\author{
Marcos Mariño and Tomás Reis \\ Département de Physique Théorique et Section de Mathématiques, Université de Genève, \\ Genève, CH-1211 Switzerland \\ E-mail: marcos.marino@unige.ch, tomas.reis@unige.ch
}

ABSTRACT: According to standard lore, perturbative series of super-renormalizable theories have only instanton singularities. In this paper we show that two-dimensional scalar theories with a spontaneously broken $O(N)$ symmetry at the classical level, which are super-renormalizable, have an IR renormalon singularity at large $N$. Since perturbative expansions in these theories are made around the "false vacuum" in which the global symmetry is broken, this singularity can be regarded as a manifestation of the non-perturbative absence of Goldstone bosons. We conjecture that the Borel singularity in the ground state energy of the Lieb-Liniger model is a non-relativistic manifestation of this phenomenon. We also provide en passant a detailed perturbative calculation of the Lieb-Liniger energy up to two-loops, and we check that it agrees with the prediction of the Bethe ansatz.

KEYwords: $1 / N$ Expansion, Field Theories in Lower Dimensions, Nonperturbative Effects, Renormalization Regularization and Renormalons

ARXiv EPrint: 1912.06228

Open ACCEss, (c) The Authors.

Article funded by $\mathrm{SCOAP}^{3}$. 


\section{Contents}

1 Introduction 1

2 Renormalons in the two-dimensional $O(N)$ model $\quad 3$

2.1 The ground state energy at two-loops 3

2.2 Large $N$ expansion and renormalons $\quad 8$

$\begin{array}{llr}2.3 & \text { Resurgent analysis } & 16\end{array}$

2.4 Generalization to an arbitrary potential 20

3 On the Lieb-Liniger model 22

4 Conclusions $\quad 28$

\section{Introduction}

The study of the large order behavior of perturbative series in quantum theory has provided an efficient window on non-perturbative phenomena. In quantum mechanics, it was found in $[1,2]$ and in many subsequent works that this behavior is controlled by instantons, and is due to the factorial growth in the number of Feynman diagrams [3] (see e.g. [4] for a textbook introduction). In quantum field theory, the situation is more complicated, since in many theories one can find specific diagrams which grow factorially with the loop order after integration over the momenta [5-9]. These diagrams are usually called renormalon diagrams (see [10] for an extensive review). They lead to singularities in the Borel plane of the coupling constant which, following [10], we will call renormalon singularities, or renormalons for short. Depending on the region in momenta which leads to the factorial growth, one has UV or IR renormalons. In asymptotically free theories and in QED, renormalons are believed to control the large order behavior of perturbation theory. Evidence for this was found in [11, 12] in the case of Yang-Mills theory, and in [13-16] for integrable two-dimensional theories.

It is often stated in the literature that renormalons, as their name indicate, are typical of renormalizable field theories, while super-renormalizable field theories only have instanton singularities (see e.g. [7, 17, 18]). A typical example of the latter case is the two-dimensional field theory for a real scalar field $\Phi$ with Lagrangian

$$
\mathcal{L}(\Phi)=\frac{1}{2} \partial_{\mu} \Phi \partial^{\mu} \Phi-\frac{\mu^{2}}{2} \Phi^{2}-\frac{g}{4 !} \Phi^{4} .
$$

This theory is super-renormalizable. Its perturbative expansion in powers of the dimensionless coupling $\mathrm{g} / \mathrm{m}^{2}$ is factorially divergent but Borel-summable [19-22]. Its large order behavior is controlled by instanton singularities, both in the phase where $\mu^{2}>0[21,23]$, as well as in the phase where $\mu^{2}<0$ and the $\mathbb{Z}_{2}$ symmetry is spontaneously broken [22]. 
We can promote the field $\Phi$ to an $N$-dimensional vector $\boldsymbol{\Phi}=\left(\Phi_{1}, \cdots, \Phi_{N}\right)$ and consider the version of (1.1) with a global non-Abelian symmetry $O(N)$. The theory is still superrenormalizable, but the physics when $\mu^{2}<0$ is very different: a famous theorem by Coleman, Mermin and Wagner $[24,25]$ states that there are no Goldstone bosons in two dimensions, therefore the $O(N)$ symmetry can not be broken quantum-mechanically. One symptom of this situation is that, when $\mu^{2}<0$, the quantum corrections to the vacuum expectation value (vev) of $\boldsymbol{\Phi}$ are afflicted with IR divergences. In a sense, the classical vacuum in which $\langle\boldsymbol{\Phi}\rangle \neq 0$ can be regarded as a "false vacuum" in the full quantum theory. In spite of this, it was shown by Jevicki [26] that, after renormalization and an appropriate IR regularization, the perturbative expansion for the ground state energy is well-defined, and IR divergences cancel order by order in the expansion in the coupling constant. This result was extended in $[27,28]$ to all $O(N)$-invariant correlation functions.

In this paper we argue that the perturbative series for the ground state energy of the $O(N)$ theory around this false vacuum is factorially divergent and leads to a Borel singularity in the positive real axis. Therefore, the series is not Borel summable, and the Borel singularity turns out to be an IR renormalon. This is then an example of an IR renormalon singularity in a super-renormalizable theory.

In order to establish the existence of a renormalon in this theory, we consider the leading contribution to the perturbative series at large $N$, which is due to a family of bubble-like diagrams. We show that, in agreement with Jevicki's result, their contribution is finite, order by order, but it grows factorially due to the momentum integration in the IR region. Our strategy is very similar to the analysis of renormalons in QED and QCD. In these theories, one can take a large $N_{f}$ or large $\beta_{0}$ limit, respectively, in which perturbation theory is dominated by bubble chains with a renormalon behavior [10]. The renormalon singularity we find at large $N$ is very simple and it can be analysed in detail with the tools of the theory of resurgence $[4,29,30]$. It is in fact possible to calculate the exact Borel transform of the divergent series associated to the renormalon diagrams, and to determine the full trans-series incorporating non-perturbative corrections.

The motivation for this work came from the study of a non-relativistic analogue of the two-dimensional $O(N)$ scalar theory, namely, the Lieb-Liniger model [31]. This model describes a Bose gas in one spatial dimension with a delta function repulsion, and the ground state energy can be found exactly with the Bethe ansatz. In [32] we obtained analytic results for the perturbative series of the ground state energy up to very large order and we observed that it diverges factorially, leading to a Borel singularity in the positive real axis. Instanton solutions leading to this singularity do not seem to exist. However, as we will see in this paper, the perturbative expansion of the Lieb-Liniger model is very similar to the one in the $O(N)$ model (this was already pointed out in [26]). It is then natural to conjecture that the Borel singularity found in the Lieb-Liniger model is an IR renormalon, similar to the one found in the relativistic $O(N)$ theory. We have not been able to provide a concrete realization of this scenario by finding an appropriate family of renormalon diagrams, but we go through the exercise of computing the ground state energy at two-loops by using the field theory approach of [33]. We show that the IR divergences in the Lieb-Liniger model have the same structure than the ones in the relativistic $O(N)$ 
theory, and we verify that they cancel up to two-loops. As an aside, we check that the final result agrees with the answer obtained from the Bethe ansatz. ${ }^{1}$

This paper is organized as follows. In section 2, after reviewing the results of [26], we present our main calculation, namely, the ground state energy of the $O(N)$ model at next-to-leading order in the $1 / N$ expansion. We show that the resulting expansion in the coupling constant leads to a factorially divergent series and an IR renormalon, and we perform a detailed resurgent analysis of the series. We also generalize the result to any potential for the scalar field. In section 3 we study the Lieb-Liniger model up to two-loops by using field theory techniques. Finally, in 4 we conclude and list some problems for the future.

\section{Renormalons in the two-dimensional $O(N)$ model}

\subsection{The ground state energy at two-loops}

In this paper we will focus on the perturbative series for the ground state energy of the two-dimensional $O(N)$ model. A good starting point for our analysis is a review of the two-loop calculation due to Jevicki [26] in the case of a quartic potential, since our main calculation is a large $N$ generalization of Jevicki's result. In these calculations, one performs an expansion around the classical vacuum in which the symmetry is spontaneously broken. As a consequence of the Coleman-Mermin-Wagner theorem, this is a "false vacuum" in the full quantum theory. Jevicki showed that, although IR divergences appear in intermediate steps of the calculation, they cancel in the final answer.

Let us then consider the standard $O(N)$ theory for a vector field $\boldsymbol{\Phi}=\left(\Phi_{1}, \cdots, \Phi_{N}\right)$, described by the Lagrangian

$$
\mathcal{L}(\boldsymbol{\Phi})=\frac{1}{2} \partial_{\mu} \boldsymbol{\Phi} \cdot \partial^{\mu} \boldsymbol{\Phi}-V(\boldsymbol{\Phi}),
$$

where the potential is given by

$$
V(\boldsymbol{\Phi})=\frac{\mu^{2}}{2} \boldsymbol{\Phi}^{2}+\frac{g}{4 !} \boldsymbol{\Phi}^{4}
$$

We denote $\boldsymbol{\Phi}^{4}=\left(\boldsymbol{\Phi}^{2}\right)^{2}$. We will consider the stable case in which $g>0$. Classically, there are two phases. When $\mu^{2}>0$, the absolute minimum occurs at $\boldsymbol{\Phi}=0$. This is the symmetric phase. The phase of spontaneously broken symmetry corresponds to

$$
\mu^{2}<0
$$

In this case, the minimum occurs at

$$
\Phi^{2}=\phi_{\star}^{2}=-3 ! \frac{\mu^{2}}{g} .
$$

Classically, the $O(N)$ symmetry is spontaneously broken down to $O(N-1)$.

\footnotetext{
${ }^{1}$ The agreement with the Bethe ansatz up to two-loops was verified long ago in the very different hydrodynamic formalism of $[34,35]$, which does not have IR divergences.
} 


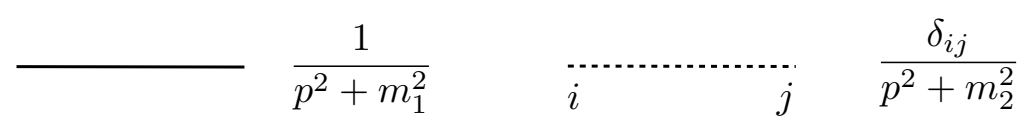

Figure 1. The propagators for the $\xi$ and the $\boldsymbol{\eta}$ fields.

Quantum mechanically, to determine the ground state and the ground state energy, we have to compute the Coleman-Weinberg effective potential [36]. We will follow [37]: first we split $\boldsymbol{\Phi}$ as

$$
\mathbf{\Phi}=(\phi, 0, \cdots, 0)+(\xi, 0, \cdots, 0)+\left(0, \eta_{1}, \cdots, \eta_{N-1}\right),
$$

where $\phi$ is a constant field configuration, while $\xi, \boldsymbol{\eta}=\left(\eta_{1}, \cdots, \eta_{N-1}\right)$ are quantum fluctuations. We will calculate $V(\phi)$ as the sum of all one-particle irreducible diagrams. The quantum corrected vev $\phi$ is then determined by

$$
\frac{\partial V}{\partial \phi}=0
$$

and the ground state energy is obtained by evaluating the effective potential on the solution of (2.6). From now on we will work in the Euclidean theory. The relevant Lagrangian is

$$
\begin{aligned}
\mathcal{L}(\phi, \xi, \boldsymbol{\eta})= & \frac{1}{2} \mu^{2} \phi^{2}+\frac{g}{4 !} \phi^{4} \\
& +\frac{1}{2} \partial_{\mu} \xi \partial^{\mu} \xi+\frac{m_{1}^{2}}{2} \xi^{2}+\frac{1}{2} \partial_{\mu} \boldsymbol{\eta} \cdot \partial^{\mu} \boldsymbol{\eta}+\frac{m_{2}^{2}}{2} \boldsymbol{\eta}^{2} \\
& +\frac{g}{3 !} \phi \xi^{3}+\frac{g}{3 !} \phi \xi \boldsymbol{\eta}^{2}+\frac{g}{12} \xi^{2} \boldsymbol{\eta}^{2}+\frac{g}{4 !} \xi^{4}+\frac{g}{4 !} \boldsymbol{\eta}^{4} .
\end{aligned}
$$

We have removed the linear vertex in $\xi$, following the prescription of [37]. In the second line of (2.7) we have introduced the masses

$$
m_{1}^{2}=\mu^{2}+\frac{g}{2} \phi^{2}, \quad m_{2}^{2}=\mu^{2}+\frac{g \phi^{2}}{3 !}+\epsilon^{2} .
$$

Following Jevicki [26], we have introduced an IR regulator $\epsilon^{2}$ which will be taken to zero at the end of the calculations. When evaluated at the classical minimum (2.4), we have

$$
m_{1}^{2}=-2 \mu^{2}:=m^{2}, \quad m_{2}^{2}=\epsilon^{2} .
$$

In the limit $\epsilon \rightarrow 0$, the $N-1$ scalar fields $\eta_{i}, i=1, \cdots, N-1$ become massless and are the classical Goldstone bosons of the model. The propagator of the $\xi$ field is represented by a full line, while the one of the $\boldsymbol{\eta}$ fields is represented a dashed line; they are shown in figure 1.

Finally, the third line in (2.7) gives the interaction terms. This leads to five types of Feynman vertices which we represent in figure 2.

In order to keep track of the loop order we introduce a $\hbar$ parameter so that the effective potential reads, up to two-loops,

$$
V\left(\phi^{2}\right)=\frac{1}{\hbar} V_{0}\left(\phi^{2}\right)+V_{1}\left(\phi^{2}\right)+\hbar V_{2}\left(\phi^{2}\right)+\cdots
$$




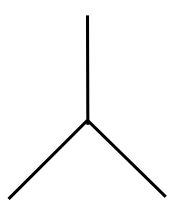

$-\frac{g}{3 !} \phi$

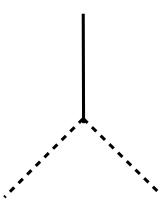

$-\frac{g}{3 !} \phi$

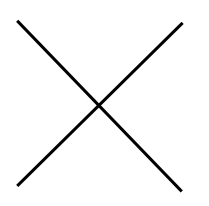

$-\frac{g}{4 !}$

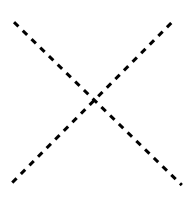

$-\frac{g}{4 !}$

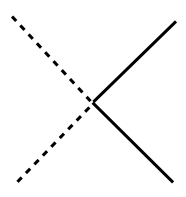

$-\frac{g}{12}$

Figure 2. The vertices for the spontaneously broken phase of the $O(N)$ model.

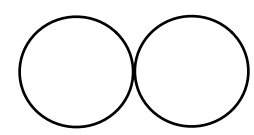

(a)

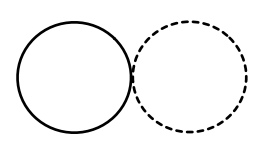

(b)

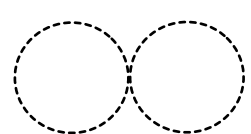

(c)

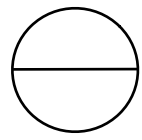

$(d)$

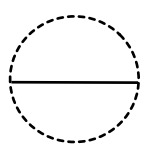

$(e)$

Figure 3. Diagrams contributing to the two-loop effective potential.

The tree-level and one-loop contributions are given by

$$
\begin{aligned}
& V_{0}(\phi)=\frac{1}{2} \mu^{2} \phi^{2}+\frac{g}{4 !} \phi^{4} \\
& V_{1}(\phi)=\frac{1}{2} \int \frac{\mathrm{d}^{d} k}{(2 \pi)^{d}} \log \left(k^{2}+m_{1}^{2}\right)+\frac{1}{2}(N-1) \int \frac{\mathrm{d}^{d} k}{(2 \pi)^{d}} \log \left(k^{2}+m_{2}^{2}\right) .
\end{aligned}
$$

The two-loop contribution is given by the sum of the diagrams in figure 3 . We will write it as

$$
V_{2}(\phi)=V_{2}^{(1)}(\phi)+V_{2}^{(2)}(\phi)
$$

where $V_{2}^{(1)}$ only involves products of one-loop integrals, while $V_{2}^{(2)}$ involves genuine twoloop integrals. We have,

$$
V_{2}^{(1)}=(a)+(b)+(c),
$$

and

$$
\begin{aligned}
& (a)=3 \frac{g}{4 !}\left(\int \frac{\mathrm{d}^{d} k}{(2 \pi)^{d}} \frac{1}{k^{2}+m_{1}^{2}}\right)^{2}, \\
& (b)=2(N-1) \frac{g}{4 !}\left(\int \frac{\mathrm{d}^{d} k}{(2 \pi)^{d}} \frac{1}{k^{2}+m_{1}^{2}}\right)\left(\int \frac{\mathrm{d}^{d} k}{(2 \pi)^{d}} \frac{1}{k^{2}+m_{2}^{2}}\right), \\
& (c)=\left(N^{2}-1\right) \frac{g}{4 !}\left(\int \frac{\mathrm{d}^{d} k}{(2 \pi)^{d}} \frac{1}{k^{2}+m_{2}^{2}}\right)^{2} .
\end{aligned}
$$

On the other hand,

$$
V_{2}^{(2)}=(d)+(e)
$$


where

$$
\begin{aligned}
& (d)=-\frac{3 !}{2}\left(\frac{g}{3 !}\right)^{2} \phi^{2} \int \frac{\mathrm{d}^{d} k}{(2 \pi)^{d}} \frac{\mathrm{d}^{d} l}{(2 \pi)^{d}} \frac{1}{k^{2}+m_{1}^{2}} \frac{1}{l^{2}+m_{1}^{2}} \frac{1}{(k+l)^{2}+m_{1}^{2}}, \\
& (e)=-\left(\frac{g}{3 !}\right)^{2} \phi^{2}(N-1) \int \frac{\mathrm{d}^{d} k}{(2 \pi)^{d}} \frac{\mathrm{d}^{d} l}{(2 \pi)^{d}} \frac{1}{k^{2}+m_{2}^{2}} \frac{1}{l^{2}+m_{2}^{2}} \frac{1}{(k+l)^{2}+m_{1}^{2}} .
\end{aligned}
$$

We now solve the minimization equation (2.6) order by order in the loop expansion, as

$$
\phi^{2}=\phi_{\star}^{2}+\hbar \phi_{1}^{2}+\hbar^{2} \phi_{2}^{2}+\cdots,
$$

where $\phi_{\star}^{2}$ is the classical value (2.4). The first quantum correction is

$$
\phi_{1}^{2}=-\left(\frac{\partial^{2} V_{0}}{\partial\left(\phi^{2}\right)^{2}}\left(\phi_{\star}^{2}\right)\right)^{-1} \frac{\partial V_{1}}{\partial \phi^{2}}\left(\phi_{\star}^{2}\right) .
$$

The final result for the ground state energy at two-loops is

$$
E\left(m^{2}, g\right)=\frac{1}{\hbar} V_{0}\left(\phi_{\star}^{2}\right)+V_{1}\left(\phi_{\star}^{2}\right)+\hbar\left(V_{2}\left(\phi_{\star}^{2}\right)-\frac{1}{2} \frac{\partial^{2} V_{0}}{\partial\left(\phi^{2}\right)^{2}}\left(\phi_{\star}^{2}\right) \phi_{1}^{4}\right)+\cdots
$$

So far we have not evaluated the Feynman integrals, which display both UV divergences as well as IR divergences when the IR regulator $\epsilon$ is taken to zero. UV divergences in scalar field theories in two dimensions can be renormalized by normal-ordering the fields. In the case of an $N$-dimensional vector, one has

$$
\begin{aligned}
& : \boldsymbol{\Phi}^{2}:=\boldsymbol{\Phi}^{2}-N \mathcal{I}(\mathrm{m}), \\
& : \boldsymbol{\Phi}^{4}:=\boldsymbol{\Phi}^{4}-2(N+2) \mathcal{I}(\mathrm{m}) \boldsymbol{\Phi}^{2}+N(N+2) \mathcal{I}^{2}(\mathrm{~m}),
\end{aligned}
$$

where

$$
\mathcal{I}(\mathrm{m})=\int \frac{\mathrm{d}^{d} k}{(2 \pi)^{d}} \frac{1}{k^{2}+\mathrm{m}^{2}},
$$

and $\mathrm{m}$ parametrizes the choice of mass in the normal ordering. Different choices of $\mathrm{m}$ correspond to different renormalization schemes. We note that $\mathrm{m}$ is not necessarily the mass of the scalar field appearing in the theory. Normal-ordering renormalization is equivalent to renormalizing the mass and the vacuum energy in the original Lagrangian (2.1) (see the recent discussions in $[21,22,38,39])$. The mass renormalization is

$$
\mu^{2}=\tilde{\mu}^{2}-g \frac{N+2}{6} \mathcal{I}(\mathrm{m}),
$$

where $\mu$ is the bare mass and $\tilde{\mu}$ is the renormalized mass. In addition, one has to add to the vacuum energy the counterterm

$$
g \frac{N(N+2)}{4 !} \mathcal{I}^{2}(\mathrm{~m})
$$

One property of normal ordering is that, if there is an interacting scalar field of mass $\mathrm{m}$, diagrams involving contractions of legs in the same vertex vanish. Therefore, a convenient 
choice, made in [26], is to do normal ordering w.r.t. the mass of the $\xi$ field in the classical vacuum, i.e. to choose

$$
\mathrm{m}^{2}=m^{2}
$$

This is the scheme also used in the recent works [21, 22]. In practice, this means that the UV divergent one-loop integrals appearing in the calculations above are renormalized as

$$
\int \frac{\mathrm{d}^{2} k}{(2 \pi)^{2}} \frac{1}{k^{2}+a^{2}} \rightarrow \int \frac{\mathrm{d}^{2} k}{(2 \pi)^{2}}\left(\frac{1}{k^{2}+a^{2}}-\frac{1}{k^{2}+m^{2}}\right)=-\frac{1}{4 \pi} \log \left(\frac{a^{2}}{m^{2}}\right) .
$$

With this choice of renormalization scheme, the diagrams (a) and (b) vanish. We renormalize the one-loop effective potential (i.e. the vacuum energy) in a way which is consistent with (2.25), namely

$$
\int \frac{\mathrm{d}^{2} k}{(2 \pi)^{2}} \log \left(k^{2}+a^{2}\right) \rightarrow \frac{a^{2}}{4 \pi}\left(1-\log \left(\frac{a^{2}}{m^{2}}\right)\right) .
$$

In particular, (2.11) reads,

$$
V_{1}\left(\phi_{\star}^{2}\right)=\frac{m^{2}}{8 \pi}+\frac{N-1}{8 \pi} \epsilon^{2}\left(1-\log \left(\frac{\epsilon^{2}}{m^{2}}\right)\right) .
$$

All UV divergences are removed by this procedure. However, we still have to take care of possible IR divergences as we send the IR cutoff to zero: $\epsilon^{2} \rightarrow 0$. Some of the quantities we have computed are genuinely IR divergent, like for example the one-loop correction to the vev,

$$
\phi_{1}^{2}=\frac{N-1}{4 \pi} \log \left(\frac{\epsilon^{2}}{m^{2}}\right) .
$$

This is a reflection of the Coleman-Mermin-Wagner theorem, namely the classical vev is destabilized by IR divergences in the quantum corrections. However, Jevicki observed that when the IR regulated results are plugged into (2.19), the IR divergences cancel and the limit $\epsilon^{2} \rightarrow 0$ leads to a finite result. To see this, we note that the Feynman integral appearing in the diagram (e) is given by

$$
\int \frac{\mathrm{d}^{2} k}{(2 \pi)^{2}} \frac{\mathrm{d}^{2} l}{(2 \pi)^{2}} \frac{1}{k^{2}+\epsilon^{2}} \frac{1}{l^{2}+\epsilon^{2}} \frac{1}{(k+l)^{2}+m^{2}}=\frac{1}{(4 \pi m)^{2}}\left(\left(\log \left(\frac{\epsilon^{2}}{m^{2}}\right)\right)^{2}+\frac{\pi^{2}}{3}\right)+\mathcal{O}\left(\epsilon^{2}\right) .
$$

It is now easy to see that the IR divergences of the form $\log ^{2}\left(\epsilon^{2}\right)$ appearing in $V_{2}\left(\phi_{\star}^{2}\right)$ cancel against the term involving $\phi_{1}^{4}$ in the ground-state energy. Finally, the integral appearing in (d) is finite and given by (see e.g. [40])

$$
\int \frac{\mathrm{d}^{2} k}{(2 \pi)^{2}} \frac{\mathrm{d}^{2} l}{(2 \pi)^{2}} \frac{1}{k^{2}+m^{2}} \frac{1}{l^{2}+m^{2}} \frac{1}{(k+l)^{2}+m^{2}}=\frac{1}{(4 \pi)^{2}} \frac{2 A}{m^{2}}
$$

where

$$
A=\sqrt{3} \operatorname{ImLi}_{2}\left(\mathrm{e}^{2 \pi \mathrm{i} / 3}\right)
$$


Putting all together, we finally obtain Jevicki's result [26]

$$
\mathcal{E}(\widehat{g}, N)=-\frac{3}{8 \widehat{g}}+\frac{1}{8 \pi}-\left(\frac{A}{2}+\frac{N-1}{12} \frac{\pi^{2}}{3}\right) \frac{\widehat{g}}{(4 \pi)^{2}}+\mathcal{O}\left(\widehat{g}^{2}\right),
$$

where we have used the dimensionless quantities

$$
\widehat{g}=\frac{g}{m^{2}}, \quad \mathcal{E}=\frac{E}{m^{2}} .
$$

The well-defined series (2.32) is expected to give the correct asymptotic expansion for the ground state energy of the theory $(2.1)$ with $\mu^{2}<0$. As already noted by Jevicki in [26], this is confirmed by a non-relativistic analogue of this theory with an $O(2)$ global symmetry: the Lieb-Liniger model [31]. In that model, one calculates the perturbative series for the ground state energy by expanding around a classical vacuum which breaks the $O(2)$ symmetry. The resulting series is not only well-defined, but it gives the correct asymptotic expansion of the ground state energy, as it can be verified directly by comparing it to the weak-coupling expansion of the exact solution obtained with the Bethe ansatz. We will perform this detailed check and comparison up to two-loops in our study of the Lieb-Liniger model in section 3.

\section{$2.2 \quad$ Large $N$ expansion and renormalons}

The ground state energy density, which is given at two-loops in (2.32), depends on $N$ and $\hat{g}$. By using large $N$ counting, it is easy to show that its perturbative expansion in powers of $\hat{g}$ has the following structure,

$$
\mathcal{E}(\widehat{g}, N)=-\frac{3}{8 \widehat{g}}+\sum_{k \geq 0}\left(\sum_{r=1}^{1+k} e_{r, k} N^{1+k-r}\right) \widehat{g}^{k} .
$$

In particular, each coefficient in this series is a polynomial in $N$. The calculation of the full perturbative series (2.34) to high loop order is difficult, but one can consider the limit in which $N$ is large. In this case, at each order in $\hat{g}$, the leading contribution comes from the coefficients $e_{1, k}$. As we will see, this sequence of coefficients is associated to a very specific type of diagrams and it can be calculated in closed form. It grows factorially with $k$, and leads to a Borel singularity in the positive real axis. This implies the existence of a renormalon singularity in the original perturbative series, at least if $N$ is large enough. This strategy to study the renormalon structure of $\mathcal{E}(\widehat{g}, N)$ is similar to the large $N_{f}$ or large $\beta_{0}$ limit of the perturbative expansion in QED and QCD, respectively. In this limit, perturbation theory is dominated by bubble diagrams, and this can be used to establish the existence of renormalons in these theories [10]. Renormalons in the scalar $O(N)$ theory in four dimensions have been also studied by calculating the effective potential at next-toleading order in the large $N$ expansion [41].

In order to further understand the structure of (2.34) at large $N$, it is convenient to introduce the 't Hooft coupling

$$
\lambda=g N
$$


and its dimensionless counterpart,

$$
\widehat{\lambda}=\widehat{g} N
$$

and reorganize the expansion as

$$
\mathcal{E}(\widehat{g}, N)=\sum_{r \geq 0} N^{1-r} \mathcal{E}_{(r)}(\widehat{\lambda}),
$$

where

$$
\mathcal{E}_{(0)}(\widehat{\lambda})=-\frac{3}{8 \widehat{\lambda}}
$$

and

$$
\mathcal{E}_{(r)}(\widehat{\lambda})=\sum_{k \geq 0} e_{r, k} \widehat{\lambda}^{k}
$$

In the large $N$ limit at fixed 't Hooft coupling, the first non-trivial contribution to the energy is given by $\mathcal{E}_{(1)}$, which encodes the coefficients $e_{1, k}$ for all $k$. From the point of view of the $1 / N$ expansion, this is the next-to-leading contribution to the energy density, and it can be calculated from the $1 / N$ expansion of the effective potential.

The leading large $N$ contribution to the effective potential in dimensions $1 \leq d \leq 4$ was obtained in the well-known paper [42], and the subleading corrections were discussed in [43]. Let us then review the relevant large $N$ techniques developed in [42, 43]. The first step is to perform a Hubbard-Stratonovich transformation. This leads to an equivalent theory with two fields, $\boldsymbol{\Phi}$ and $X$, and Lagrangian,

$$
\begin{aligned}
\mathcal{L}(\boldsymbol{\Phi}, X) & =\mathcal{L}(\boldsymbol{\Phi})+\frac{3 N}{2 \lambda}\left(X-\frac{\lambda}{6 N} \boldsymbol{\Phi}^{2}-\mu^{2}\right)^{2} \\
& =\frac{1}{2} \partial_{\mu} \boldsymbol{\Phi} \cdot \partial^{\mu} \boldsymbol{\Phi}-\frac{X}{2} \boldsymbol{\Phi}^{2}+\frac{3 N}{2 \lambda} X^{2}-\frac{3 N \mu^{2}}{\lambda} X+\frac{3 N \mu^{4}}{2 \lambda} .
\end{aligned}
$$

This theory is equivalent to the original one since $X$ is an auxiliary field which can be integrated out. To obtain the effective potential, we expand around a constant configuration of the fields,

$$
\Phi_{1}(x)=\xi(x)+\sqrt{N} \phi, \quad X(x)=\chi+\frac{\tilde{\chi}(x)}{\sqrt{N}}, \quad \Phi_{j}(x)=\eta_{j-1}(x), \quad j=2, \cdots, N .
$$

After removing the linear terms and going to Euclidean signature, we obtain the Lagrangian

$$
\begin{aligned}
\mathcal{L}(\boldsymbol{\eta}, \xi, \tilde{\chi} ; \phi, \chi)= & V_{\text {tree }}(\phi, \chi)+\frac{1}{2} \partial_{\mu} \boldsymbol{\eta} \cdot \partial^{\mu} \boldsymbol{\eta}+\frac{1}{2} \chi \boldsymbol{\eta}^{2} \\
& +\frac{1}{2} \partial_{\mu} \xi \cdot \partial^{\mu} \xi+\frac{1}{2} \chi \xi^{2}-\frac{3 \tilde{\chi}^{2}}{2 \lambda}+\tilde{\chi} \xi \phi \\
& +\frac{1}{2 \sqrt{N}} \tilde{\chi} \xi^{2}+\frac{1}{2 \sqrt{N}} \tilde{\chi} \boldsymbol{\eta}^{2}
\end{aligned}
$$

In this expression,

$$
V_{\text {tree }}(\phi, \chi)=N\left(-\frac{3 \chi^{2}}{2 \lambda}+\frac{\chi \phi^{2}}{2}+\frac{3 \mu^{2} \chi}{\lambda}-\frac{3 \mu^{4}}{2 \lambda}\right)
$$


is the tree level contribution. The effective potential has the large $N$ expansion

$$
V(\phi, \chi)=N \sum_{k \geq 0} V_{(k)}(\phi, \chi) N^{-k}
$$

and the minimization conditions

$$
\frac{\partial V}{\partial \phi}=\frac{\partial V}{\partial \chi}=0
$$

can be also solved order by order in the $1 / N$ expansion,

$$
\phi=\phi_{(0)}+\frac{1}{N} \phi_{(1)}+\cdots, \quad \chi=\chi_{(0)}+\frac{1}{N} \chi_{(1)}+\cdots
$$

At large $N$, the effective potential is given by the tree level contribution, plus the one-loop contribution of the $N-1 \eta$ fields. We find in this way, after using the renormalization scheme discussed in section 2.1,

$$
V_{(0)}(\phi, \chi)=-\frac{3 \chi^{2}}{2 \lambda}+\frac{\chi \phi^{2}}{2}+\frac{3 \mu^{2} \chi}{\lambda}-\frac{3 \mu^{4}}{2 \lambda}+\frac{1}{8 \pi} \chi\left(1-\log \left(\frac{\chi}{m^{2}}\right)\right),
$$

which is the result of Coleman, Jackiw and Politzer [42]. ${ }^{2}$

In order to calculate the coefficients $e_{1, k}$ in (2.34), we have to expand the effective potential around the saddle point which corresponds to the conventional perturbative vacuum. The minimization conditions (2.45) give

$$
\phi_{(0)}^{2}=\frac{6 \chi_{(0)}}{\lambda}-\frac{6 \mu^{2}}{\lambda}+\frac{1}{4 \pi} \log \left(\frac{\chi_{(0)}}{m^{2}}\right)
$$

as well as

$$
\chi_{(0)}=0 .
$$

This leads to an IR divergence when plugged in (2.48), which was interpreted in [42] as a manifestation of the Coleman-Mermin-Wagner theorem. However, we can regulate this IR divergence as in [26], by setting

$$
\chi_{(0)}=\epsilon^{2}
$$

and taking the limit $\epsilon \rightarrow 0$ at the end of the calculation. By plugging this in (2.48), we find the large $N$ limit of the result (2.28) obtained in the loop expansion. Therefore, the saddle-point which makes contact with the conventional, IR-regularized perturbative expansion, is given by $(2.48),(2.50) .^{3}$

\footnotetext{
${ }^{2}$ The renormalization of the mass $(2.22)$ due to normal ordering coincides at large $N$ with the renormalization scheme used in [42].

${ }^{3}$ One could argue that the expansion should be done around the "true" vacuum at large $N$, which is located at $\phi=0$. However, such an expansion would not be connected to the perturbative expansion (2.34) around the classical vacuum, which is what we want to study here. This is similar to what happens in Fermi gases with an attractive interaction. There, one can expand around a non-trivial large $N$ vacuum with a gap, which is useful to study the theory at strong coupling, or one can expand around the perturbative vacuum, which leads to a sum over ladder diagrams [44] with renormalon behavior [45]. We would like to thank Marco Serone for illuminating conversations on this point.
} 


$$
D_{\eta_{j} \eta_{k}}=\frac{\delta_{j k}}{p^{2}+\chi}
$$

$D_{\xi \xi}$
$D_{\tilde{\chi} \tilde{\chi}}$

$D_{\tilde{\chi} \xi}$

Figure 4. The propagators for the effective Lagrangian (2.42).
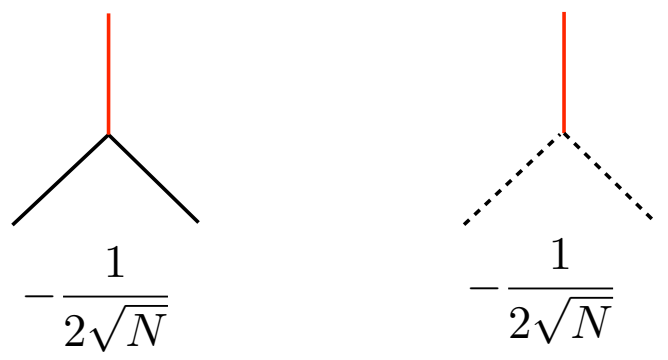

Figure 5. The vertices for the effective Lagrangian (2.42). The first one represents the $\tilde{\chi} \xi^{2}$ coupling, while the second one represents the $\tilde{\chi} \eta^{2}$ coupling.

Let us now calculate the next-to-leading correction in $1 / N$ to the effective potential, following [43]. We can think about (2.42) as a theory of the fields $\boldsymbol{\eta}, \tilde{\chi}$ and $\xi$, with a non-diagonal propagator for $\xi, \tilde{\chi}$. The inverse propagator for $\xi, \tilde{\chi}$ is given by the matrix

$$
\left(\begin{array}{cc}
p^{2}+\chi & \phi \\
\phi & -3 / \lambda
\end{array}\right)
$$

After inversion, we find the following propagators:

$$
\begin{aligned}
D_{\xi \xi} & =\frac{1}{p^{2}+\chi+\lambda \phi^{2} / 3}, \\
D_{\xi \tilde{\chi}} & =\frac{\lambda \phi}{3} \frac{1}{p^{2}+\chi+\lambda \phi^{2} / 3}, \\
D_{\tilde{\chi} \tilde{\chi}} & =-\frac{\lambda}{3} \frac{p^{2}+\chi}{p^{2}+\chi+\lambda \phi^{2} / 3} .
\end{aligned}
$$

In addition, the propagator for $\eta_{j} \eta_{k}$ is given by

$$
D_{\eta_{j} \eta_{k}}=\frac{\delta_{j k}}{p^{2}+\chi} .
$$

These propagators are represented graphically in figure 4 . The $\xi, \eta, \tilde{\chi}$ fields are represented by a full black line, dashed black line and full red line, respectively. In addition, we have the cubic vertices shown in figure 5 .

At one-loop, the $\eta$ fields gave a contribution of order $N$ in (2.47), but since there are only $N-1$ of them they also give a subleading contribution in the $1 / N$ expansion,

$$
-\frac{1}{2} \int \frac{\mathrm{d}^{d} k}{(2 \pi)^{d}} \log \left(k^{2}+\chi\right) .
$$




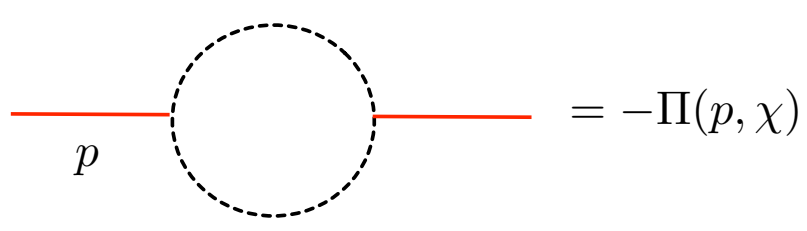

Figure 6. The polarization loop, which gives the function $\Pi(p, \chi)$ introduced in (2.56).

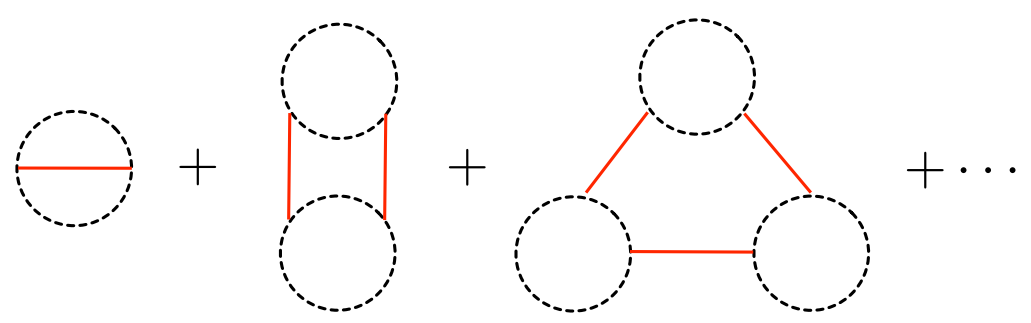

Figure 7. Ring diagrams contributing to $V_{(1)}(\phi, \chi)$.

The fields $\xi, \tilde{\chi}$ are coupled and they give the following contribution of order one to the effective potential:

$$
\frac{1}{2} \int \frac{\mathrm{d}^{d} p}{(2 \pi)^{d}} \log \left(p^{2}+\chi+\frac{\lambda \phi^{2}}{3}\right)
$$

up to an irrelevant constant.

What other diagrams can contribute at this order? It turns out that the only contribution comes from ring diagrams. The building block of ring diagrams is the polarization loop $\Pi(p, \chi)$, which is shown in figure 6 and given by

$$
\Pi(p, \chi)=\frac{1}{2} \int \frac{\mathrm{d}^{2} q}{(2 \pi)^{d}} \frac{1}{\left(q^{2}+\chi\right)\left((p+q)^{2}+\chi\right)} .
$$

Note that this is of order one at large $N$. We can now join $n$ copies of this loop through $n$ propagators of the $\tilde{\chi}$ field, as shown in figure 7 . The sum of these ring diagrams gives the following contribution to the effective potential,

$$
-\sum_{n \geq 1} \frac{1}{2 n} \int \frac{\mathrm{d}^{d} p}{(2 \pi)^{d}}\left(D_{\chi \chi} \Pi(p, \chi)\right)^{n}=-\sum_{n \geq 1} \frac{1}{2 n} \int \frac{\mathrm{d}^{d} p}{(2 \pi)^{d}}\left(-\frac{\lambda}{3} \frac{\left(p^{2}+\chi\right) \Pi(p, \chi)}{p^{2}+\chi+\lambda \phi^{2} / 3}\right)^{n} .
$$

This can be summed in closed form to obtain

$$
\frac{1}{2} \int \frac{\mathrm{d}^{d} k}{(2 \pi)^{d}} \log \left[\frac{\left(k^{2}+\chi\right)(1+\lambda \Pi(k, \chi) / 3)+\lambda \phi^{2} / 3}{k^{2}+\chi+\lambda \phi^{2} / 3}\right] .
$$

Together with $(2.55)$ we obtain

$$
V_{(1)}(\phi, \chi)=\frac{1}{2} \int \frac{\mathrm{d}^{d} k}{(2 \pi)^{d}} \log \left[\frac{\left(k^{2}+\chi\right)(1+\lambda \Pi(k, \chi) / 3)+\lambda \phi^{2} / 3}{k^{2}+\chi}\right] .
$$


We can now use these results to compute the ground state energy. By evaluating the effective potential at its minimum we find

$$
\begin{aligned}
E= & N V_{(0)}\left(\phi_{(0)}^{2}, \chi_{(0)}\right)+\frac{\partial V_{(0)}}{\partial \phi^{2}}\left(\phi_{(0)}^{2}, \chi_{(0)}\right) \phi_{(1)}^{2}+\frac{\partial V_{(0)}}{\partial \chi}\left(\phi_{(0)}^{2}, \chi_{(0)}\right) \chi_{(1)} \\
& +V_{(1)}\left(\phi_{(0)}^{2}, \chi_{(0)}\right)+\mathcal{O}\left(\frac{1}{N}\right) .
\end{aligned}
$$

Due to the minimization conditions, the terms involving $\phi_{(1)}^{2}, \chi_{(1)}$ in the first line of (2.60) in principle vanish. One has to be careful, however, due to the regularization of IR divergences: since

$$
\frac{\partial V_{(0)}}{\partial \phi^{2}}=\chi_{(0)}=\epsilon^{2}
$$

we have to calculate the limit $\epsilon \rightarrow 0$ of the second term in the first line of (2.60) explicitly. By using the explicit expression for $\phi_{(1)}^{2}$ it can be checked that indeed

$$
E=N V_{(0)}\left(\phi_{(0)}^{2}, \chi_{(0)}\right)+V_{(1)}\left(\phi_{(0)}^{2}, \chi_{(0)}\right)+\mathcal{O}\left(\frac{1}{N}\right)
$$

The leading term is indeed given by (2.38). By using (2.59) and (2.48), and taking into account our renormalization scheme, we obtain

$$
\mathcal{E}_{(1)}=\frac{1}{8 \pi}+\frac{1}{2 m^{2}} \int \frac{\mathrm{d}^{2} k}{(2 \pi)^{2}} \log \left[1+\frac{\lambda}{3} \frac{1}{k^{2}+m^{2}}\left(\left(k^{2}+\epsilon^{2}\right) \Pi\left(k, \epsilon^{2}\right)+\frac{1}{4 \pi} \log \left(\frac{\epsilon^{2}}{m^{2}}\right)\right)\right] .
$$

As emphasized in [46], the IR divergences for the ground state energy have to cancel order by order in the $1 / N$ expansion. It is now possible to check explicitly the absence of IR singularities in $\mathcal{E}_{(1)}$. The polarization loop can be computed explicitly (see e.g. appendix A in [4]):

$$
\Pi(p, \chi)=\frac{1}{4 \pi \sqrt{p^{2}\left(p^{2}+4 \chi\right)}} \log \frac{\sqrt{p^{2}+4 \chi}+\sqrt{p^{2}}}{\sqrt{p^{2}+4 \chi}-\sqrt{p^{2}}} .
$$

In the limit $\chi=\epsilon^{2} \rightarrow 0$ we find

$$
\Pi\left(p, \epsilon^{2}\right)=\frac{1}{4 \pi p^{2}} \log \left(\frac{p^{2}}{\epsilon^{2}}\right)+\mathcal{O}\left(\epsilon^{2}\right) .
$$

This is indeed IR divergent, but the divergence cancels against the contribution due to $\phi_{(0)}^{2}$. In the $\epsilon^{2} \rightarrow 0$ limit, one finds

$$
\mathcal{E}_{(1)}=\frac{1}{8 \pi}+\frac{1}{2 m^{2}} \int \frac{\mathrm{d}^{2} k}{(2 \pi)^{2}} \log \left[1+\frac{\lambda}{12 \pi} \frac{1}{k^{2}+m^{2}} \log \left(\frac{k^{2}}{m^{2}}\right)\right] .
$$

This expression is IR finite but UV divergent, due to the linear term in $\lambda$. The UV divergence can be removed by using our renormalization scheme. This is easier to do in the original representation (2.63), or by requiring that we reproduce the two-loop result (2.32) 
at this order in the $1 / N$ expansion. In this way, we obtain the manifestly finite, surprisingly simple answer for the subleading correction to the ground state energy in the $1 / N$ expansion,

$$
\mathcal{E}_{(1)}=\frac{1}{8 \pi}-\frac{\pi}{48} \gamma-\mathcal{I}(\gamma)
$$

where $\mathcal{I}(\gamma)$ is the integral

$$
\mathcal{I}(\gamma)=\frac{1}{8 \pi} \int_{0}^{\infty} \mathrm{d} x\left\{\log \left[1+\gamma \frac{\log (x)}{x+1}\right]-\gamma \frac{\log (x)}{x+1}\right\}
$$

and $\gamma$ is the dimensionless coupling

$$
\gamma=\frac{\widehat{\lambda}}{12 \pi}
$$

It is useful to expand the function $\mathcal{I}(\gamma)$ in powers of $\gamma$ :

$$
\mathcal{E}_{(1)} \sim \frac{1}{8 \pi}-\frac{\pi}{48} \gamma-\sum_{n=0}^{\infty} c_{n} \gamma^{n+2} .
$$

The coefficients $c_{n}$ are given by

$$
c_{n}=\frac{(-1)^{n}}{8 \pi(n+2)} \int_{0}^{\infty}\left(\frac{\log (x)}{1+x}\right)^{n+2} \mathrm{~d} x, \quad n \geq 0 .
$$

The integrals can be computed as derivatives of the beta function, as in a similar calculation in [47]:

$$
\int_{0}^{\infty} \frac{\mathrm{d} x}{(x+1)^{n}}(\log (x))^{n}=\left.(-1)^{n} \frac{\mathrm{d}^{n}}{\mathrm{~d} z^{n}} B(1-z, n-1+z)\right|_{z=0} .
$$

Of course, the coefficients $e_{1, n+2}$ appearing in (2.34), (2.39) are given, up to a overall factor $(12 \pi)^{-n-2}$, by the $c_{n}$ in (2.71). We have then determined the leading contribution to the perturbative expansion (2.34) when $N$ is large.

What is the large order behavior of the $c_{n}$ ? Let us first note that their integrand involves the $n$-th power of the logarithm of the momentum, which is typical of renormalon diagrams [10]. As $n$ grows, this integrand has larger and larger values near $k=0$, i.e. in the IR region, while the UV behavior as $k \rightarrow \infty$ is tamed by the denominator $\left(k^{2}+m^{2}\right)^{n}$. It is indeed easy to show that ${ }^{4}$

$$
c_{n} \sim \frac{n !}{8 \pi}, \quad n \gg 1
$$

Therefore, the first non-trivial contribution to the ground state energy in the $1 / N$ expansion is given by a factorially divergent series in the coupling constant. The factorial growth is due to the integration over momenta in the IR region, and leads to a singularity in the Borel plane of the dimensionless coupling constant $\widehat{g}$. The singularity is located, at large $N$, at

$$
\zeta=\frac{12 \pi}{N}
$$

\footnotetext{
${ }^{4}$ In the next section we will derive a closed formula for the $c_{n}$, as well as a precise asymptotic expansion for them.
} 
Therefore, this is an IR renormalon. It leads to a non-perturbative ambiguity in this theory, characterized by the exponentially small scale

$$
\exp \left(-\frac{12 \pi}{N \widehat{g}}\right)
$$

Since the singularity is in the positive real axis, the perturbative expansion in this theory is not Borel summable, for $N>1$. This is in contrast to the case $N=1$, where the series is Borel summable [19-22]. It is interesting to note that the argument in [20-22] for Borel summability can not be applied in the case of two-dimensional theories with a continuum of degenerate vacua, which is precisely the case we are looking at.

Let us make some additional comments on this result:

1. Although the coupling constant $g$ does not get renormalized, the mass does, and this leads to a renormalization of the dimensionless coupling constant $\widehat{g}$. The corresponding beta function at one-loop is

$$
\beta(\widehat{g})=-\frac{N+2}{6 \pi} \widehat{g}^{2}
$$

The coefficient appearing in the exponent of (2.75) can then be interpreted as twice the coefficient of this beta function, at large $N$. This is similar to what happens with standard IR renormalons in an asymptotically free theory.

2. A similar calculation can be done in the theory (2.1) but in three dimensions, which is also super-renormalizable. In this case, the symmetry remains broken quantummechanically [42] and the classical vacuum is the "true" vacuum at weak coupling. The $1 / N$ correction to the ground state energy leads to a series which grows only exponentially and has a finite radius of convergence, which is the expected behavior in the absence of renormalons (see e.g. [4, 29]). This supports our interpretation of the IR renormalon in (2.70) as a manifestation of the Coleman-Mermin-Wagner theorem.

3. As in the case of QED or QCD renormalons, the IR renormalon at (2.74) is associated to bubble-like diagrams, which in the Hubbard-Stratonovich form of the theory (2.40) are the ring diagrams of figure 7 . In order to cancel IR divergences, however, one also has to add the one-loop contribution (2.55). In the original formulation of the theory, the factorially divergent behavior is not due to a single class of diagrams but is the result of different types of diagrams that have to be combined in order to obtain an IR finite result. The large $N$ expansion finds the right combination of diagrams for us.

4. The original integral form (2.67) provides a resummation of the power series (2.70), but it gives a complex result, for any value of $\lambda$. The imaginary part is of order (2.75). This is yet another manifestation of the lack of Borel summability of the original series, as we will make more precise in the next section. 
5. It is interesting to note that the non-perturbative scale (2.75) shows up when this model is studied on a two-dimensional Anti-de Sitter space [48, 49]. In $\mathrm{AdS}_{2}$ the Coleman-Mermin-Wagner theorem can be evaded and, if the $\mathrm{AdS}_{2}$ radius $R$ is sufficiently small, there is a phase where the $O(N)$ symmetry is spontaneously broken. The boundary separating this phase from the phase with unbroken symmetry is given by [48]

$$
R M=\exp \left(-\frac{12 \pi}{N \widehat{g}}-\gamma_{E}\right)
$$

where $M$ is a renormalization mass scale (we note that in [48] the UV divergences are regularized by a cut-off). This involves precisely the non-perturbative ambiguity (2.75).

6. In [22], complex instantons were found for the theory (1.1) with $\mu^{2}<0$ and with a single scalar field (i.e. $N=1$ ). These solutions to the classical EOM can be trivially embedded in the $O(N)$ theory we have studied, and we expect them to lead to singularities in the Borel plane. Singularities associated to complex instantons do not obstruct Borel summability, but they might control the leading large order behavior of the perturbative series for some values of $N$, similarly to what happens in [50].

7. There might be additional instanton singularities in this theory, and one could ask whether the singularity (2.74) can be associated to an instanton. Usually, factorial growth in the loop expansion at a fixed order in the $1 / N$ expansion can not be justified by instanton singularities (see e.g. [13, 29]). In addition, it is easy to see that any real solution to the Euclidean EOM has a negative real action,

$$
S=-\frac{g}{4 !} \int \mathrm{d}^{d} x \boldsymbol{\Phi}^{4}(x),
$$

therefore an instanton configuration leading to the singularity at (2.74) has to be complex but lead to a positive, real action. Moreover, this action should scale as $1 / N$ for large $N$. In some models, and after a twisted compactification, such scaling can be obtained through fractional instantons [51-57]. However, in this case, and in infinite volume, an instanton interpretation of the singularity (2.74) seems difficult to achieve.

8. The structure of (2.67) is very similar to the $1 / N$ correction to the ground state energy of the attractive Gaudin-Yang model with $N$ components, calculated in [45]. In both cases, ring diagrams diverge factorially and they are resummed by a logarithm which gives an exponentially small imaginary part. In the case of the Gaudin-Yang model, this imaginary part is a manifestation of the Cooper instability.

\subsection{Resurgent analysis}

As we have seen in the previous section, the series (2.70), which is obtained from ring diagrams, diverges factorially and leads to a singularity in the Borel plane. Factorially 
divergent series arising in the large $N_{f}$ limit of QED (or the large $\beta_{0}$ limit of QCD) can sometimes be analyzed in great detail, and their Borel transforms computed in closed form, see [10] for examples. In this section we will perform such an analysis for (2.70) by using the theory of resurgence (for this example, we will only need the tools presented in [4]).

Let us consider the formal power series appearing in (2.70),

$$
\varphi(\gamma)=\sum_{n=0}^{\infty} c_{n} \gamma^{n},
$$

as well as its Borel transform

$$
\widehat{\varphi}(\zeta)=\sum_{n=0}^{\infty} \frac{c_{n}}{n !} \zeta^{n}
$$

This Borel transform has singularities on the positive real axis, so the conventional Borel resummation is not well-defined. However, we can define the lateral Borel resummations as

$$
s_{ \pm}(\varphi)(z)=z^{-1} \int_{\mathcal{C}_{ \pm}} \mathrm{d} \zeta \mathrm{e}^{-\zeta / z} \widehat{\varphi}(\zeta)
$$

where $\mathcal{C}_{ \pm}$are integration paths slightly above (respectively, below) the positive real axis. These lateral Borel resummations have an imaginary piece which, according to the theory of resurgence, can be obtained by an appropriate resummation of a formal trans-series, involving both the coupling $\gamma$ and the exponentially small coupling $\mathrm{e}^{-1 / \gamma}$. We expect this trans-series to be of the form

$$
\sum_{\ell=1}^{\infty} C_{\ell} \mathrm{e}^{-\ell / \gamma} \gamma^{-b_{\ell}} \varphi_{\ell}(\gamma),
$$

where $C_{\ell}$ are constants (sometimes called the trans-series parameters) and

$$
\varphi_{\ell}(z)=\sum_{n \geq 0} a_{\ell, n} \gamma^{n}
$$

is a, in general divergent, formal power series in $\gamma$.

It turns out that in this example the trans-series (2.82) can be computed in a simple way. The reason is the following. The resummed energy can be written in terms of the integral $\mathcal{I}(\gamma)$ in (2.68). As we mentioned in the previous section, this integral has an imaginary part which is easy to calculate. For any value of $\gamma$, the argument of the logarithm becomes negative for

$$
0<x<x(\gamma)
$$

where $x(\gamma)$ is the solution to the equation

$$
1+\gamma \frac{\log (x)}{x+1}=0
$$

Along the interval $[0, x(\gamma)]$, the integrand of (2.68) has a constant imaginary part, given by $\pm \mathrm{i} / 8$, where the sign depends on a choice of sign for $\log (-1)= \pm \pi \mathrm{i}$. The imaginary part of $\mathcal{I}(\gamma)$ is then given by

$$
\operatorname{Im} \mathcal{I}(\gamma)= \pm \frac{\mathrm{i}}{8} x(\gamma)
$$




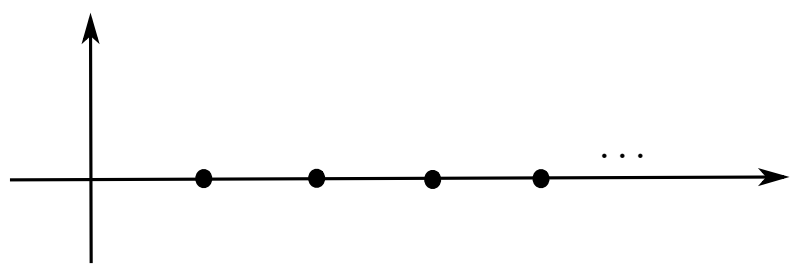

Figure 8. The Borel transform (2.80) has poles of order $\ell+1$ at all positive integers $\ell=1,2, \cdots$. The $\ell$-th singularity leads to an exponentially small correction $\exp (-12 \pi \ell /(N \widehat{g}))$ in the trans-series.

We expect this imaginary part to agree (up to an overall factor $\gamma^{2}$ ) with the imaginary part of the lateral Borel resummations (2.81). On the other hand, an explicit solution to (2.85) can be found by using Lambert's function $W(x)$ :

$$
x(\gamma)=\gamma W\left(\gamma^{-1} \mathrm{e}^{-1 / \gamma}\right)=\sum_{\ell=1}^{\infty} \frac{\ell^{\ell-1}}{\ell !}(-1)^{\ell-1} \gamma^{1-\ell} \mathrm{e}^{-\ell / \gamma} .
$$

From this we can read the trans-series $(2.82)$ associated to $\varphi(\gamma)$ : it has

$$
b_{\ell}=\ell+1, \quad \varphi_{\ell}(\gamma)=\frac{1}{8} \frac{\ell^{\ell-1}}{\ell !}(-1)^{\ell-1} .
$$

In this case, the series $\varphi_{\ell}(\gamma)$ truncate to a single coefficient (this truncation seems to be typical of trans-series appearing in large $N$ renormalon calculations). Note in addition that, as a series in $\mathrm{e}^{-1 / \gamma} / \gamma$, the trans-series has a finite radius of convergence. This also happens in other examples (see e.g. [58, 59]). One can verify numerically that the integral $\mathcal{I}(\gamma)$ agrees with the lateral Borel resummation, i.e.

$$
\mathcal{I}(\gamma)=\gamma^{2} s_{ \pm}(\varphi)(\gamma)
$$

where the choice of lateral resummation corresponds to the choice of sign in the imaginary part of $\mathcal{I}(\gamma)$.

It follows from the general theory of resurgence that the trans-series contains information about the singularities of the Borel transform of the original series. In particular, a pole of order $k$ at $\zeta=A$ in the Borel transform $\widehat{\varphi}(\zeta)$, with coefficient $a$, leads to a term in the trans-series of the form

$$
\frac{\pi a(-1)^{k-1}}{(k-1) !} \frac{\mathrm{e}^{-A / \gamma}}{\gamma^{k}}
$$

In our case, we find poles at all positive integers $\zeta=\ell$, of order $\ell+1$. More precisely, we can write

$$
\widehat{\varphi}(\zeta)=\frac{1}{8 \pi} \sum_{\ell=1}^{\infty} \frac{\ell^{\ell-1}}{(\zeta-\ell)^{\ell+1}}+\text { regular. }
$$

This gives the precise positions of all the IR renormalon singularities, which we show schematically in figure 8. We note that there are no other singularities in the Borel plane. In particular, there are no UV renormalons. The regular part in (2.91) can be guessed by 
using the fact that the coefficients $c_{n}$ only involve zeta functions evaluated at even integers. We then conjecture the following exact expression,

$$
\widehat{\varphi}(\zeta)=\frac{1}{8 \pi} \sum_{\ell=1}^{\infty}\left\{\frac{\ell^{\ell-1}}{(\zeta-\ell)^{\ell+1}}+(-1)^{\ell-1} \ell^{-\ell-1}(\zeta+\ell)^{\ell-1}\right\},
$$

which we have checked by expanding around $\zeta=0$ at very large order. Interestingly, we can now reconstruct the original perturbative coefficients in (2.80) by the Cauchy formula

$$
\frac{c_{k}}{k !}=\frac{1}{2 \pi \mathrm{i}} \oint_{\mathcal{C}_{0}} \frac{\widehat{\varphi}(\zeta)}{\zeta^{k+1}} \mathrm{~d} \zeta
$$

where $\mathcal{C}_{0}$ is a small circle around $\zeta=0$. We deform the contour $\mathcal{C}_{0}$ to pick all the poles in the positive real axis, plus a contour at infinity $\mathcal{C}_{\infty}$. We obtain in this way

$$
c_{k}=-k ! \sum_{\ell=1}^{\infty} \operatorname{Res}_{\zeta=\ell}\left(\frac{\widehat{\varphi}(\zeta)}{\zeta^{k+1}}\right)+\frac{k !}{2 \pi \mathrm{i}} \oint_{\mathcal{C}_{\infty}} \frac{\widehat{\varphi}(\zeta)}{\zeta^{k+1}} \mathrm{~d} \zeta .
$$

The sum in the r.h.s. of (2.94) gives the contribution of Borel singularities to the large order behavior of the perturbative series. By using (2.92) we find

$$
\frac{1}{8 \pi} \sum_{\ell=1}^{\infty} \ell^{-k-\ell-1} \Gamma(k+\ell+1) \frac{(-1)^{\ell-1} \ell^{\ell-1}}{\ell !}=\frac{1}{8 \pi} \sum_{\ell=1}^{\infty}(-1)^{\ell-1} \ell^{-k-2}(1+\ell)_{k},
$$

where $(\alpha)_{\beta}$ is the Pochhammer symbol. The expression in the l.h.s. has the standard form of a resurgent asymptotic formula. The contribution of the integral around infinity in (2.94) gives

$$
\frac{1}{8 \pi} \sum_{\ell=1}^{\infty}(-1)^{\ell-1} \frac{(k-\ell)(k-1-\ell) \cdots(1-\ell)}{(-\ell)^{k+2}}
$$

and we obtain

$$
c_{k}=\frac{1}{8 \pi} \sum_{\ell=1}^{\infty}(-1)^{\ell-1} \ell^{-k-2}\left((1+\ell)_{k}+(-1)^{k}(1-\ell)_{k}\right) .
$$

This can be written even more explicitly by expressing the Pochhammer symbols in terms of Stirling numbers of the first kind $s(k, n)$. We find,

$$
c_{k}=\frac{1}{4 \pi} \sum_{t=0}^{\left[\frac{k+1}{2}\right]} s(k+1, k+1-2 t)\left(1-2^{-1-2 t}\right) \zeta(2 t+2) .
$$

This provides a much more explicit expression for the coefficients of the perturbative series than the original integral formula (2.71). Therefore, in this case, knowledge of the transseries eventually leads to a better understanding of the perturbative series. Interestingly, the appearance of zeta functions in the perturbative coefficients is closely related to the sum over the singularities of the Borel transform at positive integer points.

Let us note that, if we are interested in an asympotic expansion of $c_{k}$ at large $k$, the second term in the r.h.s. of (2.97) (which comes from the contour integral at infinity) does 
not contribute, since $(1-\ell)_{k}=0$ for any $k \geq l$. Therefore, as an asymptotic expansion, we have

$$
c_{k} \sim \frac{1}{8 \pi} \sum_{\ell=1}^{\infty} \ell^{-k-\ell-1} \Gamma(k+\ell+1) \frac{(-1)^{\ell-1} \ell^{\ell-1}}{\ell !}, \quad k \gg 1 .
$$

In particular, the leading asymptotics comes from the first singularity at $\ell=1$,

$$
c_{k} \sim \frac{1}{8 \pi} \Gamma(k+2), \quad k \gg 1,
$$

with no corrections of order $1 / k$ (the corrections to this formula are exponentially small, of order $2^{-k}$ ).

\subsection{Generalization to an arbitrary potential}

The result (2.67) for the ground state energy at subleading order in $1 / N$ can be easily generalized to a scalar theory with an arbitrary potential $U(x)$, described by the Lagrangian

$$
\mathcal{L}(\boldsymbol{\Phi})=\frac{1}{2} \partial_{\mu} \boldsymbol{\Phi} \cdot \partial^{\mu} \boldsymbol{\Phi}-N U\left(\boldsymbol{\Phi}^{2} / N\right) .
$$

At the classical level, we have spontaneous symmetry breaking if the equation

$$
U^{\prime}\left(\phi^{2}\right)=0
$$

has non-trivial positive solutions. We will assume that this is the case, and we will denote by

$$
\phi_{\star}^{2}
$$

the value of $\boldsymbol{\Phi}^{2} / N$ at the minimum. At tree level we find a massive particle $\xi$ with square mass

$$
m^{2}=4 \phi_{\star}^{2} U^{\prime \prime}\left(\phi_{\star}^{2}\right)
$$

and $N-1$ Goldstone bosons $\boldsymbol{\eta}$.

The calculation of the effective potential of (2.101) at large $N$ was done in [60], while the next-to-leading correction was calculated in [61]. They generalize the quartic case considered in $[42,43]$ as well as the sextic case considered in $[62,63]$. We will present a simpler derivation, by combining ingredients from [18, 43, 64]. The first step, following [18], is to introduce two new scalar fields in the path integral, $X$ and $\Sigma$, by the following delta function trick:

$$
1=\int \mathcal{D} \Sigma \delta\left(\Sigma-\boldsymbol{\Phi}^{2} / N\right)=\int \mathcal{D} \Sigma \mathcal{D} X \exp \left\{\mathrm{i} N X / 2\left(\Sigma-\boldsymbol{\Phi}^{2} / N\right)\right\}
$$

This leads to an equivalent theory with Lagrangian

$$
\mathcal{L}(\boldsymbol{\Phi}, X, \Sigma)=\frac{1}{2} \partial_{\mu} \boldsymbol{\Phi} \cdot \partial^{\mu} \boldsymbol{\Phi}-\frac{X}{2} \boldsymbol{\Phi}^{2}-N U(\Sigma)+\frac{N \Sigma X}{2} .
$$

As usual we expand around the constant configuration

$$
\begin{aligned}
& \Phi_{1}(x)=\sqrt{N} \phi+\xi(x), \quad \Phi_{j}(x)=\eta_{j-1}(x), \quad j=2, \cdots, N, \\
& X(x)=\chi+\tilde{\chi}(x) / \sqrt{N}, \quad \Sigma=\sigma+\tilde{\sigma}(x) / \sqrt{N} .
\end{aligned}
$$


We then obtain

$$
\begin{aligned}
\mathcal{L}(\boldsymbol{\Phi}, X, \Sigma)= & -N\left(U(\sigma)-\frac{\sigma \chi}{2}+\frac{\chi \phi^{2}}{2}\right)+\frac{1}{2} \partial_{\mu} \boldsymbol{\eta} \cdot \partial^{\mu} \boldsymbol{\eta}-\frac{1}{2} \chi \boldsymbol{\eta}^{2} \\
& +\frac{1}{2} \partial_{\mu} \xi \cdot \partial^{\mu} \xi-\frac{1}{2} \chi \xi^{2}-\tilde{\chi} \xi \phi-\frac{1}{2} U^{\prime \prime}(\sigma) \tilde{\sigma}^{2}+\frac{1}{2} \tilde{\chi} \tilde{\sigma} \\
& -\frac{1}{2 \sqrt{N}} \tilde{\chi} \xi^{2}-\frac{1}{2 \sqrt{N}} \tilde{\chi} \boldsymbol{\eta}^{2} .
\end{aligned}
$$

As in previous calculations, it is more convenient to rotate to Euclidean signature. The $N-1$ scalars lead to a one-loop correction

$$
\frac{N-1}{2} \int \frac{\mathrm{d}^{d} k}{(2 \pi)^{d}} \log \left(k^{2}+\chi\right)
$$

and the final result for the effective potential at leading order in the $1 / N$ expansion is

$$
V_{(0)}(\sigma, \chi, \phi)=U(\sigma)-\frac{\sigma \chi}{2}+\frac{\chi \phi^{2}}{2}+\frac{1}{2} \int \frac{\mathrm{d}^{d} k}{(2 \pi)^{d}} \log \left(k^{2}+\chi\right) .
$$

We can compare this result to the one obtained in [60,61]. To do this, we solve for $\chi, \sigma$, by using the minimization conditions

$$
\begin{aligned}
& \frac{\partial V_{(0)}}{\partial \sigma}=U^{\prime}(\sigma)-\frac{\chi}{2}=0, \\
& \frac{\partial V_{(0)}}{\partial \chi}=\frac{\phi^{2}}{2}-\frac{\sigma}{2}+\frac{1}{2} B_{1}(\chi),
\end{aligned}
$$

where

$$
B_{1}(\chi)=\int \frac{\mathrm{d}^{d} k}{(2 \pi)^{d}} \frac{1}{k^{2}+\chi}
$$

We then solve

$$
\sigma=\phi^{2}+B_{1}(\chi), \quad \chi=2 U^{\prime}\left(\phi^{2}+B_{1}(\chi)\right) .
$$

The second equation defines $\chi$ implicitly. We conclude that

$$
V_{(0)}\left(\phi^{2}\right)=U\left(\phi^{2}+B_{1}(\chi)\right)-B_{1}(\chi) U^{\prime}\left(\phi^{2}+B_{1}(\chi)\right)+\frac{1}{2} \int \frac{\mathrm{d}^{d} k}{(2 \pi)^{d}} \log \left(k^{2}+\chi\right) .
$$

This is precisely the result obtained in $[60,61]$.

We can now calculate the next-to-leading term in $1 / N$ as we did in the quartic case, following [43]. The inverse propagator for the three fields, $\xi, \tilde{\chi}, \sigma$, is given by the matrix

$$
D^{-1}=\left(\begin{array}{ccc}
p^{2}+\chi & \phi & 0 \\
\phi & 0 & -\frac{1}{2} \\
0 & -\frac{1}{2} & U^{\prime \prime}(\sigma)
\end{array}\right) .
$$

From this we obtain the $\tilde{\chi} \tilde{\chi}$ propagator,

$$
D_{\tilde{\chi} \tilde{\chi}}(p)=-4 U^{\prime \prime}(\sigma) \frac{p^{2}+\chi}{p^{2}+\chi+4 U^{\prime \prime}(\sigma) \phi^{2}}
$$


The final result is

$$
V_{(1)}(\sigma, \chi, \phi)=\frac{1}{2} \int \frac{\mathrm{d}^{d} k}{(2 \pi)^{d}} \log \left[\frac{\left(k^{2}+\chi\right)\left(1+4 U^{\prime \prime}(\sigma) \Pi(k, \chi)\right)+4 U^{\prime \prime}(\sigma) \phi^{2}}{k^{2}+\chi}\right],
$$

in agreement with $[61,63]$.

Let us now calculate the vacuum energy at this order. The solution of $(2.113)$ is

$$
\sigma=\phi^{2}+B_{1}(\chi)=\phi_{\star}^{2}+\mathcal{S}(\chi),
$$

where $\mathcal{S}(\chi) \rightarrow 0$ when $\chi \rightarrow 0$. The function appearing in (2.117) then reads, as $\chi \rightarrow 0$,

$$
\begin{aligned}
& \left(k^{2}+\chi\right)\left(1+4 U^{\prime \prime}(\sigma) \Pi(k, \chi)\right)+4 U^{\prime \prime}(\sigma) \phi^{2} \\
& \quad=k^{2}+m^{2}+4 U^{\prime \prime}\left(\phi_{\star}^{2}\right)\left\{\left(k^{2}+\chi\right) \Pi(k, \chi)+\frac{1}{4 \pi} \log \left(\frac{\chi}{m^{2}}\right)\right\}+\mathcal{O}(\chi),
\end{aligned}
$$

where we have renormalized $B_{1}(\chi)$ by using the prescription (2.25) (this is equivalent, at large $N$, to renormalizing the coefficients of the potential, which is in turn equivalent to normal-ordering [60]). Like before, IR divergences cancel, and we find that $\mathcal{E}_{(1)}$ involves the series

$$
-\sum_{n \geq 0} c_{n}\left(\frac{1}{4 \pi \phi_{\star}^{2}}\right)^{n+2},
$$

where the coefficients $c_{n}$ are the same ones (2.71) appearing in the theory with a quartic potential. The non-perturbative ambiguity is now of the form

$$
\exp \left(-4 \pi \phi_{\star}^{2}\right) .
$$

\section{On the Lieb-Liniger model}

A Bose gas at zero temperature with chemical potential $\mu$ and a repulsive $\delta$-function interaction is described by the following action,

$$
S(\Phi)=\int \mathrm{d} t \int \mathrm{d}^{D} x\left\{\Phi^{\dagger}\left(\mathrm{i} \partial_{t}+\frac{\nabla^{2}}{2 m}+\mu\right) \Phi-\frac{1}{4} g\left(\Phi^{\dagger} \Phi\right)^{2}\right\}
$$

where $\Phi$ is a complex scalar field. The grand potential $\Omega(\mu)$ of this gas can be calculated by computing first the effective potential $\mathcal{V}(\mu, \phi)$, as a function of the vev of $\Phi, \phi$. The value of $\phi$ is then fixed by the minimization condition

$$
\frac{\partial \mathcal{V}}{\partial \phi}(\mu, \phi)=0
$$

Finally, $\Omega(\mu)$ is obtained by evaluating $\mathcal{V}(\mu, \phi)$ at this minimum.

When $D=1$, the model described by (3.1) is integrable and known as the Lieb-Liniger model [31]. In this case $\Omega(\mu)$ can be calculated with the Bethe ansatz. It has a perturbative expansion in powers of the coupling constant, with the following structure

$$
\frac{g}{\mu^{2}} \Omega(\mu)=-\sum_{n \geq 0} c_{n} \xi^{n},
$$


where

$$
\xi=\frac{g}{4 \mu^{1 / 2}}
$$

Up to order $g$, one finds [32, 34, 65]

$$
\Omega(\mu)=-\frac{\mu^{2}}{g}-\frac{\sqrt{2}}{3 \pi} \mu^{3 / 2}+\frac{g \mu}{4}\left(\frac{1}{12}-\frac{1}{\pi^{2}}\right)+\cdots
$$

In [32] we calculated the first fifty coefficients of the expansion (3.3), and we found numerically the following asymptotics

$$
c_{n} \sim A^{-n} n !, \quad A=4 \sqrt{2} \pi .
$$

In particular, the perturbative series is not Borel summable.

As we mentioned in the Introduction, there are two possible sources for the behavior (3.6): it could be due to an instanton configuration, and then $A>0$ is identified with the action of an instanton, or it could be due to renormalon diagrams. Let us first consider instantons. These are solutions of the Euclidean equations of motion (EOM) for the action (3.1), and they are given by (see e.g. [66])

$$
\begin{gathered}
\left\{\frac{\partial}{\partial \tau}-\frac{\nabla^{2}}{2 m}-\mu+\frac{g}{2}|\Phi|^{2}\right\} \Phi=0, \\
\left\{-\frac{\partial}{\partial \tau}-\frac{\nabla^{2}}{2 m}-\mu+\frac{g}{2}|\Phi|^{2}\right\} \Phi^{\dagger}=0 .
\end{gathered}
$$

It is easy to see that any solution to the above equations leads to an Euclidean action given by

$$
-\frac{g}{4} \int \mathrm{d} \tau \mathrm{d}^{D} x\left(\Phi^{\dagger} \Phi\right)^{2}
$$

similarly to (2.78). Therefore, if $\Phi^{\dagger}$ and $\Phi$ are complex conjugates, the action is negative and such an instanton, even if it exists, can not explain the Borel singularity in the positive real axis. In principle, one could consider more general instanton solutions in which $\Phi$ is not the complex conjugate of $\Phi^{\dagger}$, but we have not found any solution to the EOM which leads to a positive, real action. ${ }^{5}$ It is therefore natural to suspect that the Borel singularity associated to the large order behavior (3.6) is rather a renormalon singularity.

In fact, the Lieb-Liniger model is in many ways a non-relativistic avatar of the $O(N)$ model that we studied in the previous section. For example, in $D=1$, the field $\Phi$ in (3.1) cannot have a vev quantum-mechanically, due to the non-relativistic version of the Coleman-Mermin-Wagner theorem. However, in the standard perturbative approach to the interacting Bose gas, we expand around such a "false vacuum". As in the example studied by Jevicki and reviewed in section 2.1, we expect to have IR divergences which eventually cancel in the calculation of observables. In view of this, it is natural to conjecture that the perturbation theory of the Lieb-Liniger mode has an IR renormalon, as in the $O(N)$ model, explaining in this way the large order behavior (3.6).

\footnotetext{
${ }^{5}$ We have benefitted from many discussions with Peter Wittwer on this issue.
} 
We will now analyze the perturbative structure of the Lieb-Liniger model up to twoloops. Such an analysis was performed long ago by using a collective variable formulation [35] and Popov's hydrodynamic formalism [34] (see [67]). As emphasized in [68], this formalism is manifestly IR finite. However, it has other important drawbacks; for example, it contains an infinite number of interaction vertices. Therefore, and specially in view of further explorations of the perturbative structure, we will use the field-theoretic approach of [33], which considered the three-dimensional case $D=3$, and we will study the model in $D=1$ (the case $D=2$ was analyzed in [69], and a useful review can be found in [70]). Many aspects of this analysis are independent of the dimension, and we refer to these references for further details.

We first expand the field $\Phi$ around a constant configuration $\phi$ which breaks the global $\mathrm{U}(1)$ symmetry:

$$
\Phi=\phi+\frac{\xi+\mathrm{i} \eta}{\sqrt{2}}
$$

where $\xi, \eta$ are real fields and $\phi$ is a real positive constant. The action becomes

$$
S(\Phi)=S(\phi)+S_{\text {free }}(\xi, \eta)+S_{\mathrm{int}}(\phi, \xi, \eta) .
$$

Here,

$$
S(\phi)=V T\left(\mu \phi^{2}-\frac{1}{4} g \phi^{4}\right)
$$

is the action evaluated at $\Phi=\phi$. The free part of the action consists of the terms quadratic in $\xi$ and $\eta$ :

$$
S_{\text {free }}(\xi, \eta)=\int \mathrm{d} t \mathrm{~d}^{D} x\left\{\frac{1}{2}(\eta \dot{\xi}-\xi \dot{\eta})+\frac{1}{4 m} \xi\left(\nabla^{2}-2 m g \phi^{2}+X\right) \xi+\frac{1}{4 m} \eta\left(\nabla^{2}+X\right) \eta\right\},
$$

where

$$
X=2 m\left(\mu-\frac{1}{2} g \phi^{2}\right)
$$

From $S_{\text {free }}$ we can read the propagator for the fields $\xi$ and $\eta$ :

$$
D(\omega, k, \phi)=\frac{\mathrm{i}}{\omega^{2}-\varepsilon^{2}(k, \phi)+\mathrm{i} \epsilon}\left(\begin{array}{cc}
\left(k^{2}-X\right) / 2 m & -\mathrm{i} \omega \\
\mathrm{i} \omega & \left(k^{2}+2 m g \phi^{2}-X\right) / 2 m
\end{array}\right),
$$

where

$$
\varepsilon^{2}(k, \phi)=\frac{1}{4 m^{2}}\left(k^{2}-X\right)\left(k^{2}+2 m g \phi^{2}-X\right) .
$$

The diagonal elements of the propagator matrix (3.14) are represented by solid lines for $\xi$ and dashed lines for $\eta$. The off-diagonal elements are represented by a line that is half solid and half dashed, as illustrated in figure 9. Finally, the interaction part of the action is given by

$$
S_{\text {int }}(\phi, \xi, \eta)=\int \mathrm{d} t \int \mathrm{d}^{D} x\left\{\frac{\phi X}{\sqrt{2} m} \xi-\frac{g \phi}{\sqrt{8}} \xi\left(\xi^{2}+\eta^{2}\right)-\frac{g}{16}\left(\xi^{2}+\eta^{2}\right)^{2}\right\} .
$$

It leads to the vertices shown in figure 10 . 
Figure 9. The propagators for the $\eta, \xi$ fields.

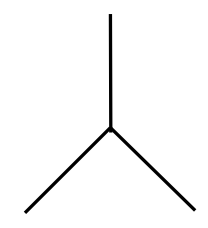

$-\frac{g \phi}{\sqrt{8}}$

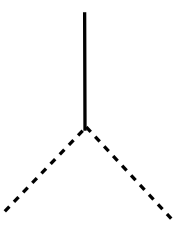

$-\frac{g \phi}{\sqrt{8}}$

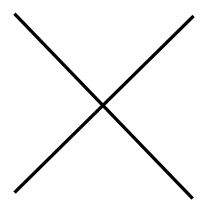

$-\frac{g}{16}$

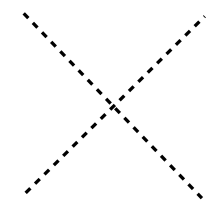

$-\frac{g}{16}$

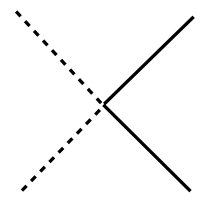

$-\frac{g}{8}$

Figure 10. The vertices for the interactions between $\eta$, $\xi$ fields.

Let us now calculate the loop expansion of $\mathcal{V}(\mu, \phi)$. The tree-level contribution can be obtained from $S(\phi)$ :

$$
\mathcal{V}_{0}(\mu, \phi)=-\mu \phi^{2}+\frac{1}{4} g \phi^{4}
$$

The one-loop contribution is

$$
\mathcal{V}_{1}(\mu, \phi)=\frac{\mathrm{i}}{2} \int \frac{\mathrm{d}^{D} k}{(2 \pi)^{D}} \int \frac{\mathrm{d} \omega}{2 \pi} \log \operatorname{det} D(\omega, k, \phi)=\frac{1}{2} \int \frac{\mathrm{d}^{D} k}{(2 \pi)^{D}} \varepsilon(k, \phi) .
$$

One-loop calculations in this theory involve the integrals

$$
\mathcal{I}_{m, n}=\int \frac{\mathrm{d}^{D} p}{(2 \pi)^{D}} \frac{\left(p^{2}-X\right)^{m}}{(2 m \varepsilon(p, \phi))^{n}}
$$

so one can write

$$
\mathcal{V}_{1}(\mu, \phi)=\frac{1}{4 m} \mathcal{I}_{0,-1}
$$

Finally, the two-loop potential $\mathcal{V}_{2}(\mu, \phi)$ is the sum of the contributions of the vacuum diagrams shown in figure 11. The diagrams in the first line involve products of one-loop integrals:

$$
\mathcal{V}_{2}^{(a)}=\frac{3}{64} g \mathcal{I}_{1,1}^{2}, \quad \mathcal{V}_{2}^{(b)}=\frac{1}{32} g \mathcal{I}_{-1,-1} \mathcal{I}_{1,1}, \quad \mathcal{V}_{2}^{(c)}=\frac{3}{64} g \mathcal{I}_{-1,-1}^{2}
$$

The diagrams in the second line involve the integrals

$$
\begin{aligned}
\mathcal{J}_{l, m, n}=\int \frac{\mathrm{d}^{D} p}{(2 \pi)^{D}} \int \frac{\mathrm{d}^{D} q}{(2 \pi)^{D}} & \left(\frac{p^{2}-X}{2 m \varepsilon(p, \phi)}\right)^{l}\left(\frac{q^{2}-X}{2 m \varepsilon(q, \phi)}\right)^{m}\left(\frac{r^{2}-X}{2 m \varepsilon(r, \phi)}\right)^{n} \\
& \times \frac{1}{2 m(\varepsilon(p, \phi)+\varepsilon(q, \phi)+\varepsilon(r, \phi))}
\end{aligned}
$$




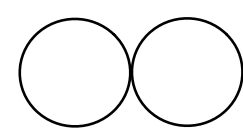

(a)

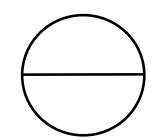

(d)

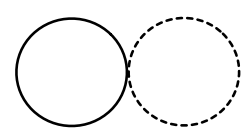

(b)

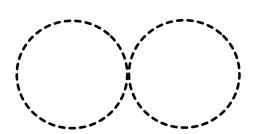

(c)

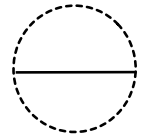

(e)

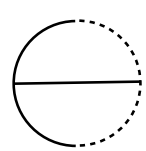

$(f)$

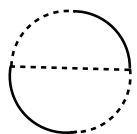

(g)

Figure 11. Two-loop diagrams for the Lieb-Liniger model.

where $r=|\mathbf{p}+\mathbf{q}|$. They are given by

$$
\begin{aligned}
\mathcal{V}_{2}^{(d)} & =-\frac{3}{16} m g^{2} \phi^{2} \mathcal{J}_{1,1,1}, & \mathcal{V}_{2}^{(e)} & =-\frac{1}{16} m g^{2} \phi^{2} \mathcal{J}_{-1,-1,1}, \\
\mathcal{V}_{2}^{(f)} & =\frac{3}{8} m g^{2} \phi^{2} \mathcal{J}_{0,0,1}, & \mathcal{V}_{2}^{(g)} & =-\frac{1}{8} m g^{2} \phi^{2} \mathcal{J}_{-1,0,0}
\end{aligned}
$$

We can now find the minimum $\phi$ solving (3.2), which we also expand according to the loop order as

$$
\phi=\phi_{\star}+\phi_{1}+\cdots
$$

The classical vev $\phi_{\star}$ minimizes (3.17) and one finds

$$
\phi_{\star}^{2}=\frac{2 \mu}{g} .
$$

For this value of $\phi$, the parameter $X$ in (3.13) vanishes. The field $\eta$ is massless and is the Goldstone boson of the model. The propagator simplifies when $X=0$ and one recovers Bogoliubov's dispersion relation:

$$
\varepsilon\left(k, \phi_{\star}\right)=\frac{k \sqrt{k^{2}+\Lambda^{2}}}{2 m},
$$

where

$$
\Lambda^{2}=4 m \mu \text {. }
$$

However, it is easy to see that when $D=1$ and $X=0$ many of the integrals above are IR divergent. We will then proceed as we have done in the relativistic case and we will introduce an explicit IR regulator by setting

$$
X=-\epsilon^{2} .
$$

We will take $\epsilon \rightarrow 0$ at the end of the calculation. Let us then introduce the regulated dispersion relation

$$
\varepsilon(k ; \epsilon)=\frac{\sqrt{k^{2}+\epsilon^{2}} \sqrt{k^{2}+\Lambda^{2}}}{2 m},
$$

the regulated one-loop integrals

$$
I_{m, n}(\Lambda, \epsilon)=\int \frac{\mathrm{d}^{D} p}{(2 \pi)^{D}} \frac{\left(p^{2}+\epsilon^{2}\right)^{m}}{(2 m \varepsilon(p ; \epsilon))^{n}},
$$


and the regulated two-loop integrals

$$
\begin{aligned}
J_{l, m, n}(\Lambda, \epsilon)=\int \frac{\mathrm{d}^{D} p}{(2 \pi)^{D}} \int \frac{\mathrm{d}^{D} q}{(2 \pi)^{D}} & \left(\frac{p^{2}+\epsilon^{2}}{2 m \varepsilon(p ; \epsilon)}\right)^{l}\left(\frac{q^{2}+\epsilon^{2}}{2 m \varepsilon(q ; \epsilon)}\right)^{m}\left(\frac{r^{2}+\epsilon^{2}}{2 m \varepsilon(r ; \epsilon)}\right)^{n} \\
& \times \frac{1}{2 m(\varepsilon(p ; \epsilon)+\varepsilon(q ; \epsilon)+\varepsilon(r ; \epsilon))} .
\end{aligned}
$$

We can now express all results in terms of these regulated integrals. The one-loop correction to the condensate is given by [33]

$$
\phi_{1}=-\frac{g \phi_{\star}}{16 \mu}\left(3 I_{1,1}+I_{-1,-1}\right),
$$

and the final result for the grand potential up to two-loops is as in [33],

$$
\Omega(\mu)=-\frac{\mu^{2}}{g}+\frac{1}{4 m} I_{0,-1}+\frac{m g \mu}{8} J+\frac{g}{32}\left(I_{-1,-1}^{2}-2 I_{-1,-1} I_{1,1}-3 I_{1,1}^{2}\right),
$$

where

$$
J(\epsilon)=6 J_{0,0,1}-J_{-1,-1,1}-3 J_{1,1,1}-2 J_{-1,0,0}
$$

and it is independent of $\Lambda$. Of course, all the integrals have to be understood as IR regularized integrals.

Let us analyze these integrals in more detail. It turns out that, in $D=1, I_{0,-1}$ and $I_{1,1}$ are IR convergent but UV divergent. After dimensional regularization they lead to finite results that can be obtained explicitly from the formulae in [33]. One finds,

$$
I_{0,-1}(\Lambda, 0)=-\frac{\Lambda^{3}}{3 \pi}, \quad I_{1,1}(\Lambda, 0)=-\frac{\Lambda}{\pi} .
$$

On the other hand, $I_{-1,-1}(\Lambda, \epsilon)$ is both UV divergent and IR divergent as $\epsilon \rightarrow 0$. It can be computed for arbitrary $\epsilon$ in dimensional regularization, and one obtains a finite result for $D=1$ and $\epsilon>0$. Its expansion as $\epsilon \rightarrow 0$ can be calculated analytically and one finds,

$$
I_{-1,-1}(\Lambda, \epsilon)=\Lambda\left\{-\frac{1}{2 \pi} \log \left(\frac{\epsilon^{2}}{\Lambda^{2}}\right)+\frac{2 \log (2)-1}{\pi}+\mathcal{O}\left(\epsilon^{2}\right)\right\}
$$

Note that $\phi_{1}$, given in (3.32), inherits this divergence, which has exactly the same functional form as in the relativistic case (2.28). It is also easy to see that the two-loop integrals $J_{-1,-1,1}$ and $J_{-1,0,0}$ are both IR divergent. The integral $J_{-1,-1,1}$ comes from the diagram (e) in figure 11, which is the counterpart of the IR divergent diagram also labelled as (e) in figure 3 . The integral $J_{-1,0,0}$ appears in the diagram (f) in figure 11. It involves the "mixed" propagator of the $\xi$ and $\eta$ fields, and it has no counterpart in the relativistic $O(N)$ theory. However, as in [26], all divergences appearing in the calculation of the grand potential should cancel order by order in the $g$ expansion. Using the values of the integrals (3.35) and (3.36), and setting $m=1 / 2$ for convenience, we find the following result for the grand potential at two loops:

$$
\begin{aligned}
\Omega(\mu)= & -\frac{\mu^{2}}{g}-\frac{\sqrt{2}}{3 \pi} \mu^{3 / 2} \\
& +\frac{\mu g}{16}\left(J(\epsilon)+\frac{1}{4 \pi^{2}} \log ^{2}\left(\epsilon^{2}\right)-\frac{2 \log (2)}{\pi^{2}} \log \left(\epsilon^{2}\right)+\frac{4 \log ^{2}(2)}{\pi^{2}}-\frac{4}{\pi^{2}}\right)+\cdots
\end{aligned}
$$


We note that the integral $J(\epsilon)$ can be further simplified and put into the form

$$
\begin{aligned}
J(\epsilon) & =\frac{1}{4 \pi^{2}} \int \mathrm{d} p \mathrm{~d} q \mathrm{~d} r \delta(p+q+r) F(p, q, r ; \epsilon) \\
& =\frac{1}{\pi^{2}} \int_{0}^{\infty} \mathrm{d} p \int_{0}^{p} \mathrm{~d} q(F(p, q, p+q ; \epsilon)+F(p, q, p-q ; \epsilon)),
\end{aligned}
$$

where

$$
F(p, q, r ; \epsilon)=-\frac{1}{3} \frac{1}{E_{p}+E_{q}+E_{r}} \frac{1}{\alpha_{p} \alpha_{q} \alpha_{r}}\left(\alpha_{p} \alpha_{q}+\alpha_{p} \alpha_{r}+\alpha_{q} \alpha_{r}-3\right)^{2},
$$

and

$$
E_{k}=\sqrt{k^{2}+1} \sqrt{k^{2}+\epsilon^{2}}, \quad \alpha_{k}=\sqrt{\frac{k^{2}+1}{k^{2}+\epsilon^{2}}}
$$

The result (3.37) agrees with (3.5) up to one-loop. In order to have agreement up to two-loops, we must have

$$
\lim _{\epsilon \rightarrow 0}\left(J(\epsilon)+\frac{1}{4 \pi^{2}} \log ^{2}\left(\epsilon^{2}\right)-\frac{2 \log (2)}{\pi^{2}} \log \left(\epsilon^{2}\right)\right)=\frac{1}{3}-\frac{4 \log ^{2}(2)}{\pi^{2}} .
$$

We have not attempted to establish (3.41) analytically. Numerically, we find

$$
\lim _{\epsilon \rightarrow 0}\left(J(\epsilon)+\frac{1}{4 \pi^{2}} \log ^{2}\left(\epsilon^{2}\right)-\frac{2 \log (2)}{\pi^{2}} \log \left(\epsilon^{2}\right)\right) \approx 1.38613061 \ldots
$$

which agrees with the r.h.s. of (3.41) in all stable numerical digits.

In order to establish the existence of an IR renormalon, one should find a calculable family of diagrams which leads to an IR finite answer and which grow factorially with the number of loops. In the case of the relativistic $O(N)$ scalar theory, the choice of such a set of diagrams was done for us by the large $N$ expansion. There is a natural $\mathrm{U}(N)$ generalization of the theory with action (3.1) (see e.g. [71-73]), but in $D=1$ the dominant and subdominant diagrams at large $N$ do not contribute to the ground state energy (as expected from [74]), and the $1 / N$ expansion is of little help in this case. It would be very interesting to complete this study by finding a sequence of diagrams which leads to an IR renormalon and explains the large order behavior (3.6).

\section{Conclusions}

In this paper we have studied super-renormalizable scalar field theories in two-dimensions with a spontaneously broken $O(N)$ symmetry at the classical level. As noted long ago by Jevicki, one can obtain an IR finite perturbative series around the classical vacuum of these theories. These series give the correct weak-coupling, asymptotic expansion of the observables. However, we have shown by an explicit large $N$ calculation that the resulting series for the ground state energy is not Borel summable and leads to an IR renormalon singularity.

This IR renormalon qualifies the assertion often found in the literature according to which there are no renormalons in super-renormalizable theories. We have argued that 
the physical reason behind this renormalon is that, as a consequence of the ColemanMermin-Wagner theorem, one is expanding around a "false vacuum". Although the IR divergences due to the would-be Goldstone bosons cancel, the perturbative series remains "IR sensitive" [10] and leads to the IR renormalon.

The $O(N)$ model can be described at low energies by the non-linear sigma model [18]. Since the latter has IR renormalons, this would explain the appearance of an IR renormalon in the former. ${ }^{6}$ It would be illuminating to make this more precise, but it seems clear that the $O(N)$ model provides a much simpler realization of IR renormalons than the non-linear sigma model, precisely due to its simpler UV behavior. In particular, we do not expect the $O(N)$ model to have UV renormalons, while observables in the non-linear sigma model display a complicated mixing of both IR and UV renormalons (see e.g. [16] for an example).

It is also interesting to compare the non-Borel summable perturbative series we find in the $O(N)$ model with the one around the perturbative vacua in the double-well potential. In quantum mechanics, the parity symmetry of this potential can not be spontaneously broken, and the perturbative vacua are false vacua. The lack of Borel summability of the resulting perturbative series reflects the wrong choice of vacuum that one has made to begin with. In this quantum-mechanical example, the factorial divergence is an instanton effect and can be cured by taking into account multi-instanton sectors (see e.g. $[75,76]$ ). In the case of the $O(N)$ theory, we are also expanding around a false vacuum, due to the Coleman-Mermin-Wagner theorem. The lack of Borel summability might be interpreted as the price to pay for committing the original sin of expanding around this incorrect vacuum. However, as we showed in this paper, the resulting Borel singularity is in this case an IR renormalon effect.

Our finding further supports the idea that renormalons should not be associated exclusively to renormalizable theories. Renormalon singularities have been recently found in different contexts, like quantum mechanics [77] and various condensed matter models [45]. In the case studied in this paper, as well as in the examples of [45], renormalon singularities are due to the integration over momenta in "dangerous" regions, which are not even necessarily the IR or UV regions (for example, in many-fermion models, these regions are often associated to the Fermi surface).

The IR renormalon unveiled in this paper is perhaps one of the simplest incarnations of a renormalon singularity. It shows that the perturbative approach to the two-dimensional $O(N)$ quantum field theory is insufficient, and one has to take into account some sort of nonperturbative effect in order to make sense of perturbation theory. Another manifestation of the simplicity of this model is that it is possible to make a very detailed analysis of its resurgent structure. In particular, the associated trans-series can be fully determined, and we have presented a conjectural form for the exact Borel transform which leads to an explicit expression for the original perturbative series in terms of even zeta functions. However, it is not clear to us what is the nature of the non-perturbative sectors that lead to the exponentially small corrections appearing in the trans-series. Finding an explicit description for them is probably the most important problem opened by this investigation.

\footnotetext{
${ }^{6}$ We would like to thank Lorenzo di Pietro and Marco Serone for pointing this out.
} 
There are other open problems that should be addressed. First of all, it would be very interesting to (dis)prove our conjecture about the existence of a similar renormalon in the Lieb-Liniger model. Note that in this case, with the help of the exact Bethe ansatz solution, one could try to construct the appropriate trans-series for the ground state energy. It would be also interesting to find other examples of super-renormalizable field theories with a Coleman-Mermin-Wagner "false vacuum" where one can study similar renormalon singularities in various observables. Since our result is based on a large $N$ analysis, it would be very useful to find an example where the large order behavior of the perturbative series at finite $N$ can be studied explicitly and it is controlled by a renormalon of this type.

Another natural line of enquiry, in view of recent work [78-80], is to understand the fate of the IR renormalon found in this paper after a (twisted) compactification on a circle. Perhaps the approach of [51-57] can be also applied in this case, and provide a semiclassical interpretation of this renormalon in a suitable compactification of the theory. It might also happen that compactification makes the renormalon disappear completely, as argued in [78]. In fact, as we mentioned above, the $O(N)$ theory on an $\mathrm{AdS}_{2}$ space of sufficiently small radius will have a vacuum where the symmetry is spontaneously broken. If our picture is correct, in that case we expect the perturbative series to be Borel summable again, with no trace of IR renormalons left. An explicit test of this expectation would be of great interest.

\section{Acknowledgments}

We would like to thank Jens Andersen, Volodymyr Pastukhov, Santi Peris, Lorenzo di Pietro, Marco Serone and Peter Wittwer for useful conversations and correspondence. We are particularly grateful to Gerald Dunne, Antal Jevicki, Santi Peris and Marco Serone for their comments on a preliminary version of this paper. This work has been supported in part by the Fonds National Suisse, subsidy 200021-175539, by the NCCR 51NF40141869 "The Mathematics of Physics" (SwissMAP), and by the ERC Synergy Grant "ReNewQuantum".

Open Access. This article is distributed under the terms of the Creative Commons Attribution License (CC-BY 4.0), which permits any use, distribution and reproduction in any medium, provided the original author(s) and source are credited.

\section{References}

[1] C.S. Lam, Behavior of very high order perturbation diagrams, Nuovo Cim. A $5 \mathbf{5}$ (1968) 258 [INSPIRE].

[2] C.M. Bender and T.T. Wu, Anharmonic oscillator. 2: A Study of perturbation theory in large order, Phys. Rev. D 7 (1973) 1620 [InSPIRE].

[3] C.M. Bender and T.T. Wu, Statistical Analysis of Feynman Diagrams, Phys. Rev. Lett. 37 (1976) 117 [INSPIRE].

[4] M. Mariño, Instantons and large N. An introduction to non-perturbative methods in quantum field theory, Cambridge University Press (2015). 
[5] D.J. Gross and A. Neveu, Dynamical Symmetry Breaking in Asymptotically Free Field Theories, Phys. Rev. D 10 (1974) 3235 [InSPIRE].

[6] B.E. Lautrup, On High Order Estimates in QED, Phys. Lett. B 69 (1977) 109 [InSPIRE].

[7] G. Parisi, Singularities of the Borel Transform in Renormalizable Theories, Phys. Lett. B 76 (1978) 65 [INSPIRE].

[8] G. Parisi, On Infrared Divergences, Nucl. Phys. B 150 (1979) 163 [inSPIRE].

[9] G. 't Hooft, Can We Make Sense Out of "Quantum Chromodynamics?", in The Whys of Subnuclear Physics, Subnuclear Series 15, A. Zichichi ed., Springer, Boston MA U.S.A. (1979), pp. 943-982 [inSPIRE].

[10] M. Beneke, Renormalons, Phys. Rept. 317 (1999) 1 [hep-ph/9807443] [INSPIRE].

[11] C. Bauer, G.S. Bali and A. Pineda, Compelling Evidence of Renormalons in QCD from High Order Perturbative Expansions, Phys. Rev. Lett. 108 (2012) 242002 [arXiv:1111.3946] [INSPIRE].

[12] G.S. Bali, C. Bauer and A. Pineda, Perturbative expansion of the plaquette to $\mathcal{O}\left(\alpha^{35}\right)$ in four-dimensional SU(3) gauge theory, Phys. Rev. D 89 (2014) 054505 [arXiv:1401.7999] [INSPIRE].

[13] V.A. Fateev, V.A. Kazakov and P.B. Wiegmann, Principal chiral field at large N, Nucl. Phys. B 424 (1994) 505 [hep-th/9403099] [InSPIRE].

$[14]$ D. Volin, From the mass gap in $O(N)$ to the non-Borel-summability in $O(3)$ and $O(4)$ $\sigma$-models, Phys. Rev. D 81 (2010) 105008 [arXiv:0904.2744] [INSPIRE].

[15] F. Bruckmann and M. Puhr, Universal Renormalons in Principal Chiral Models, Phys. Rev. D 101 (2020) 034513 [arXiv: 1906.09471] [INSPIRE].

[16] M. Mariño and T. Reis, Renormalons in integrable field theories, JHEP 04 (2020) 160 [arXiv: 1909.12134] [INSPIRE].

[17] J.-C. Le Guillou and J. Zinn-Justin eds., Large order behavior of perturbation theory, North-Holland (1990).

[18] J. Zinn-Justin, Quantum field theory and critical phenomena, Oxford University Press (2002).

[19] J.-P. Eckmann, J. Magnen and R. Sénéor, Decay properties and Borel summability for the Schwinger functions in $P(\varphi)_{2}$ theories, Commun. Math. Phys. 39 (1975) 251.

[20] E. Brézin, J.-C. Le Guillou and J. Zinn-Justin, Perturbation Theory at Large Order. 2. Role of the Vacuum Instability, Phys. Rev. D 15 (1977) 1558 [inSPIRE].

[21] M. Serone, G. Spada and G. Villadoro, $\lambda \phi^{4}$ Theory. Part I. The Symmetric Phase Beyond NNNNNNNNLO, JHEP 08 (2018) 148 [arXiv:1805.05882] [INSPIRE].

[22] M. Serone, G. Spada and G. Villadoro, $\lambda \phi_{2}^{4}$ theory. Part II. The broken phase beyond NNNN(NNNN)LO, JHEP 05 (2019) 047 [arXiv: 1901.05023] [INSPIRE].

[23] E. Brézin and G. Parisi, Critical exponents and large-order behavior of perturbation theory, J. Stat. Phys. 19 (1978) 269.

[24] S.R. Coleman, There are no Goldstone bosons in two-dimensions, Commun. Math. Phys. 31 (1973) 259 [INSPIRE]. 
[25] N.D. Mermin and H. Wagner, Absence of ferromagnetism or antiferromagnetism in one-dimensional or two-dimensional isotropic Heisenberg models, Phys. Rev. Lett. 17 (1966) 1133 [INSPIRE].

[26] A. Jevicki, On the Ground State and Infrared Divergences of Goldstone Bosons in Two-Dimensions, Phys. Lett. B 71 (1977) 327 [InSPIRE].

[27] S. Elitzur, The Applicability of Perturbation Expansion to Two-dimensional Goldstone Systems, Nucl. Phys. B 212 (1983) 501 [INSPIRE].

[28] F. David, Cancellations of Infrared Divergences in the Two-dimensional Nonlinear $\sigma$-models, Commun. Math. Phys. 81 (1981) 149 [inSPIRE].

[29] M. Mariño, Lectures on non-perturbative effects in large $N$ gauge theories, matrix models and strings, Fortsch. Phys. 62 (2014) 455 [arXiv: 1206.6272] [INSPIRE].

[30] I. Aniceto, G. Basar and R. Schiappa, A Primer on Resurgent Transseries and Their Asymptotics, Phys. Rept. 809 (2019) 1 [arXiv: 1802.10441] [INSPIRE].

[31] E.H. Lieb and W. Liniger, Exact analysis of an interacting Bose gas. 1. The General solution and the ground state, Phys. Rev. 130 (1963) 1605 [INSPIRE].

[32] M. Mariño and T. Reis, Exact perturbative results for the Lieb-Liniger and Gaudin-Yang models, J. Stat. Phys. 177 (2019) 1148 [arXiv:1905.09575] [inSPIRE].

[33] E. Braaten and A. Nieto, Quantum corrections to the energy density of a homogeneous Bose gas, Eur. Phys. J. B 11 (1999) 143.

[34] V.N. Popov, Theory of one-dimensional Bose gas with point interaction, Theor. Math. Phys. 30 (1977) 222.

[35] M. Takahashi, On the Validity of Collective Variable Description of Bose Systems, Prog. Theor. Phys. 53 (1975) 386.

[36] S.R. Coleman and E.J. Weinberg, Radiative Corrections as the Origin of Spontaneous Symmetry Breaking, Phys. Rev. D 7 (1973) 1888 [inSPIRE].

[37] R. Jackiw, Functional evaluation of the effective potential, Phys. Rev. D 9 (1974) 1686 [INSPIRE].

[38] S. Rychkov and L.G. Vitale, Hamiltonian truncation study of the $\varphi^{4}$ theory in two dimensions, Phys. Rev. D 91 (2015) 085011 [arXiv:1412.3460] [INSPIRE].

[39] G. Sberveglieri, M. Serone and G. Spada, Renormalization scheme dependence, RG flow, and Borel summability in $\phi^{4}$ Theories in $d<4$, Phys. Rev. D 100 (2019) 045008 [arXiv: 1905.02122] [INSPIRE].

[40] L. Adams, C. Bogner and S. Weinzierl, The two-loop sunrise graph with arbitrary masses, J. Math. Phys. 54 (2013) 052303 [arXiv:1302.7004] [INSPIRE].

[41] L. Galli and I. Scimemi, Renormalons in the effective potential of the vectorial $\left(\phi^{2}\right)^{2}$ model, Phys. Lett. B 408 (1997) 403 [hep-th/9612035] [InSPIRE].

[42] S.R. Coleman, R. Jackiw and H.D. Politzer, Spontaneous Symmetry Breaking in the $O(N)$ Model for Large N, Phys. Rev. D 10 (1974) 2491 [INSPIRE].

[43] R.G. Root, Effective Potential for the $O(N)$ Model to Order 1/N, Phys. Rev. D 10 (1974) 3322 [INSPIRE]. 
[44] P. Nozieres and S. Schmitt-Rink, Bose condensation in an attractive fermion gas: From weak to strong coupling superconductivity, J. Low Temp. Phys. 59 (1985) 195 [InSPIRE].

[45] M. Mariño and T. Reis, Resurgence for superconductors, J. Stat. Mech. 1912 (2019) 123102 [arXiv: 1905.09569] [INSPIRE].

[46] D.J. Amit and G.B. Kotliar, Nonlinear $\sigma$-model and $C P^{(n-1)}$ at $(2+\epsilon)$-dimensions, Nucl. Phys. B 170 (1980) 187 [INSPIRE].

[47] D.J. Broadhurst, Large $N$ expansion of QED: Asymptotic photon propagator and contributions to the muon anomaly, for any number of loops, Z. Phys. C 58 (1993) 339 [INSPIRE].

[48] T. Inami and H. Ooguri, NAmbu-Goldstone bosons in curved space-time, Phys. Lett. B 163 (1985) 101 [INSPIRE].

[49] D. Carmi, L. Di Pietro and S. Komatsu, A Study of Quantum Field Theories in AdS at Finite Coupling, JHEP 01 (2019) 200 [arXiv:1810.04185] [INSPIRE].

[50] A. Voros, The return of the quartic oscillator. The complex WKB method, Ann. Inst. Henri Poincaré Phys. Theor. 39 (1983) 211.

[51] P.C. Argyres and M. Ünsal, The semi-classical expansion and resurgence in gauge theories: new perturbative, instanton, bion, and renormalon effects, JHEP 08 (2012) 063 [arXiv:1206.1890] [INSPIRE].

[52] G.V. Dunne and M. Ünsal, Resurgence and Trans-series in Quantum Field Theory: The $\mathbb{C P}^{N-1}$ Model, JHEP 11 (2012) 170 [arXiv:1210.2423] [INSPIRE].

[53] A. Cherman, D. Dorigoni, G.V. Dunne and M. Ünsal, Resurgence in Quantum Field Theory: Nonperturbative Effects in the Principal Chiral Model, Phys. Rev. Lett. 112 (2014) 021601 [arXiv:1308.0127] [INSPIRE].

[54] A. Cherman, D. Dorigoni and M. Ünsal, Decoding perturbation theory using resurgence: Stokes phenomena, new saddle points and Lefschetz thimbles, JHEP 10 (2015) 056 [arXiv: 1403.1277] [INSPIRE].

[55] T. Misumi, M. Nitta and N. Sakai, Classifying bions in Grassmann $\sigma$-models and non-Abelian gauge theories by D-branes, Prog. Theor. Exp. Phys. 2015 (2015) $033 \mathrm{~B} 02$ [arXiv: 1409.3444] [INSPIRE].

[56] G.V. Dunne and M. Ünsal, Resurgence and Dynamics of $O(N)$ and Grassmannian $\sigma$-models, JHEP 09 (2015) 199 [arXiv: 1505.07803] [INSPIRE].

[57] T. Fujimori, S. Kamata, T. Misumi, M. Nitta and N. Sakai, Bion non-perturbative contributions versus infrared renormalons in two-dimensional $\mathbb{C} P^{N-1}$ models, JHEP 02 (2019) 190 [arXiv: 1810.03768] [INSPIRE].

[58] O. Costin and R.D. Costin, On the formation of singularities of solutions of nonlinear differential systems in antistokes directions, Invent. Math. 145 (2001) 425.

[59] M. Mariño, R. Schiappa and M. Weiss, Multi-Instantons and Multi-Cuts, J. Math. Phys. 50 (2009) 052301 [arXiv:0809.2619] [INSPIRE].

[60] H.J. Schnitzer, The 1/n Expansion of Renormalizable and Nonrenormalizable Scalar Field Theories, Nucl. Phys. B 109 (1976) 297 [INSPIRE].

[61] P. Rembiesa, 1/N Expansion for General Scalar Interactions: Nonleading Order and Application to Bounded Interactions, Phys. Rev. D 18 (1978) 1909 [INSPIRE]. 
[62] P.K. Townsend, Spontaneous Symmetry Breaking in $O(N)$ Symmetric $\varphi^{6}$ Theory in the $\frac{1}{N}$ Expansion, Phys. Rev. D 12 (1975) 2269 [Erratum ibid. 16 (1977) 533] [INSPIRE].

[63] P.K. Townsend, Consistency of the $1 / n$ Expansion for Three-Dimensional $\phi^{6}$ Theory, Nucl. Phys. B 118 (1977) 199 [INSPIRE].

[64] F. Cooper, P. Sodano, A. Trombettoni and A. Chodos, An $O(N)$ symmetric extension of the sine-Gordon equation, Phys. Rev. D 68 (2003) 045011 [hep-th/0304112] [INSPIRE].

[65] C.A. Tracy and H. Widom, On the ground state energy of the $\delta$-function Bose gas, J. Phys. A 49 (2016) 294001.

[66] J.W. Negele and H. Orland, Quantum Many-Particle Systems, Perseus (1988).

[67] V. Pastukhov, Ground-state properties of a dilute two-dimensional Bose gas, J. Low Temp. Phys. 194 (2019) 197.

[68] A. Jevicki, Perturbation Theory in Terms of Currents and Restoration of Continuous Symmetry in Two-dimensions, Nucl. Phys. B 146 (1978) 77 [InSPIRE].

[69] J.O. Andersen, Ground state pressure and energy density of an interacting homogeneous Bose gas in two dimensions, Eur. Phys. J. B 28 (2002) 389.

[70] J.O. Andersen, Theory of the weakly interacting Bose gas, Rev. Mod. Phys. 76 (2004) 599 [cond-mat/0305138] [INSPIRE].

[71] J.O. Andersen, 1/N-expansion and the dilute Bose gas beyond mean-field theory, cond-mat/0608265 [INSPIRE].

[72] F.S. Nogueira, Introduction to the field theory of classical and quantum phase transitions, arXiv: 1009.1603 [INSPIRE].

[73] O. Hryhorchak and V. Pastukhov, Large-N properties of a non-ideal Bose gas, J. Phys. A 52 (2019) 025002 [arXiv:1805.10142] [INSPIRE].

[74] C.N. Yang and Y.-Z. You, One-Dimensional w-Component Fermions and Bosons with Repulsive Delta Function Interaction, Chin. Phys. Lett. 28 (2011) 020503 [INSPIRE].

[75] J. Zinn-Justin and U.D. Jentschura, Multi-instantons and exact results I: Conjectures, WKB expansions, and instanton interactions, Annals Phys. 313 (2004) 197 [quant-ph/0501136] [INSPIRE].

[76] J. Zinn-Justin and U.D. Jentschura, Multi-instantons and exact results II: Specific cases, higher-order effects, and numerical calculations, Annals Phys. 313 (2004) 269 [quant-ph/0501137] [INSPIRE].

[77] C. Pazarbaşı and D. Van Den Bleeken, Renormalons in quantum mechanics, JHEP 08 (2019) 096 [arXiv: 1906. 07198] [INSPIRE].

[78] M.M. Anber and T. Sulejmanpasic, The renormalon diagram in gauge theories on $\mathbb{R}^{3} \times \mathbb{S}^{1}$, JHEP 01 (2015) 139 [arXiv:1410.0121] [INSPIRE].

[79] K. Ishikawa, O. Morikawa, K. Shibata, H. Suzuki and H. Takaura, Renormalon structure in compactified spacetime, Prog. Theor. Exp. Phys. 2020 (2020) 013B01 [arXiv:1909.09579] [INSPIRE].

[80] M. Yamazaki and K. Yonekura, Confinement as analytic continuation beyond infinite coupling, Phys. Rev. Res. 2 (2020) 013383 [arXiv:1911.06327] [INSPIRE]. 